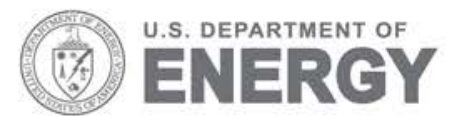

Prepared for the U.S. Department of Energy under Contract DE-AC05-76RL01830

\title{
Analysis Approach and Data Package for Mayak Public Doses
}

Paul W. Eslinger

Bruce A. Napier

August 2013 


\title{
DISCLAIMER
}

This report was prepared as an account of work sponsored by an agency of the United States Government. Neither the United States Government nor any agency thereof, nor Battelle Memorial Institute, nor any of their employees, makes any warranty, express or implied, or assumes any legal liability or responsibility for the accuracy, completeness, or usefulness of any information, apparatus, product, or process disclosed, or represents that its use would not infringe privately owned rights. Reference herein to any specific commercial product, process, or service by trade name, trademark, manufacturer, or otherwise does not necessarily constitute or imply its endorsement, recommendation, or favoring by the United States Government or any agency thereof, or Battelle Memorial Institute. The views and opinions of authors expressed herein do not necessarily state or reflect those of the United States Government or any agency thereof.

\author{
PACIFIC NORTHWEST NATIONAL LABORATORY \\ operated by \\ BATTELLE \\ for the \\ UNITED STATES DEPARTMENT OF ENERGY \\ under Contract DE-AC05-76RL01830
}

Printed in the United States of America
Available to DOE and DOE contractors from the
Office of Scientific and Technical Information,
P.O. Box 62, Oak Ridge, TN 37831-0062;
ph: (865) 576-8401
fax: (865) 576-5728
email: reports@adonis.osti.gov
Available to the public from the National Technical Information Service
5301 Shawnee Rd., Alexandria, VA 22312 ph: (800) 553-NTIS (6847)
email: orders@ntis.gov <http://www.ntis.gov/about/form.aspx>
Online ordering: http://www.ntis.gov


PNNL-22704

\section{Analysis Approach and Data package for Mayak Public Doses}

Data Supporting the Calculation of Representative Doses to Members of the Public from Airborne Releases of ${ }^{131} \mathrm{I}$ at the Mayak Production Association from 1948 through 1972

Paul W. Eslinger

Bruce A. Napier

August 2013

Prepared for

the U.S. Department of Energy

under Contract DE-AC05-76RL01830

Pacific Northwest National Laboratory

Richland, Washington 99352 


\section{SUMMARY}

Historical activities at facilities producing nuclear materials for weapons released radioactivity into the air and water. Past studies in the United States have evaluated the release, atmospheric transport and environmental accumulation of ${ }^{131}$ I from the nuclear facilities at Hanford in Washington State and the resulting dose to members of the public (Farris et al. 1994). A multi-year dose reconstruction effort (Mokrov et al. 2004) is also being conducted to produce representative dose estimates for members of the public living near Mayak, Russia, from atmospheric releases of ${ }^{131} \mathrm{I}$ at the facilities of the Mayak Production Association.

The approach to calculating individual doses to members of the public from historical releases of airborne ${ }^{131}$ I has the following general steps:

- Construct estimates of releases of ${ }^{131}$ I to the air from production facilities.

- Model the transport of ${ }^{131} \mathrm{I}$ in the air and subsequent deposition on the ground and vegetation.

- Model the accumulation of ${ }^{131} \mathrm{I}$ in soil, water and food products (environmental media).

- Calculate the dose for an individual by matching the appropriate lifestyle and consumption data for the individual to the concentrations of ${ }^{131} \mathrm{I}$ in environmental media at their residence location.

A number of computer codes were developed to facilitate the study of airborne ${ }^{131}$ I emissions at Hanford. Of particular interest is DESCARTES code that modeled accumulation of ${ }^{131} \mathrm{I}$ in environmental media (Miley et al. 1994). In addition, the CIDER computer code estimated annual doses to individuals (Eslinger et al. 1994) using the equations and parameters specific to Hanford (Snyder et al. 1994).

Several of the computer codes developed to model ${ }^{131}$ I releases from Hanford are general enough to be used for other facilities. Additional codes have been developed, including the new individual dose code CiderF (Eslinger and Napier 2013), and applied to historical releases of ${ }^{131}$ I from Mayak. This document provides a data package that identifies computer code runs and associated input and output files prepared for the purpose of calculating doses to members of the public from atmospheric releases of ${ }^{131} \mathrm{I}$ at the Mayak Production Association for the time period 1948 through 1972. 


\section{Contents}

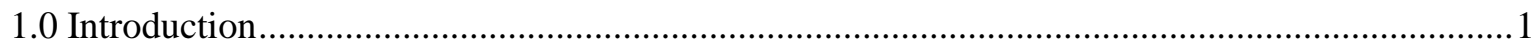

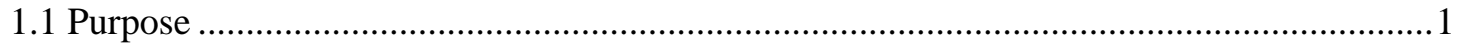

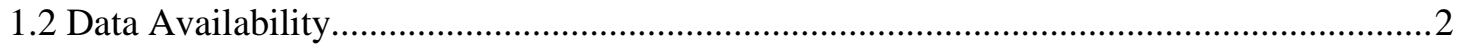

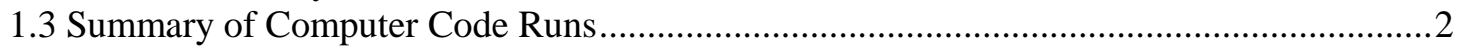

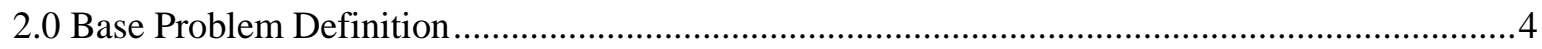

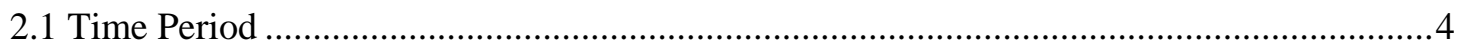

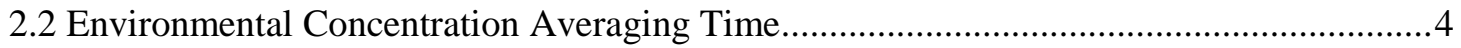

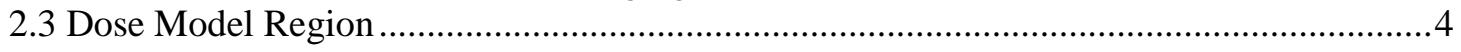

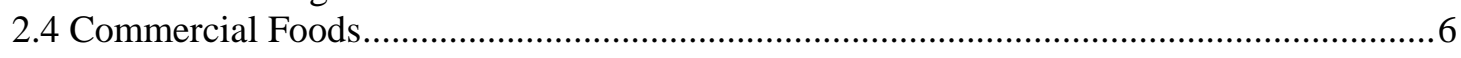

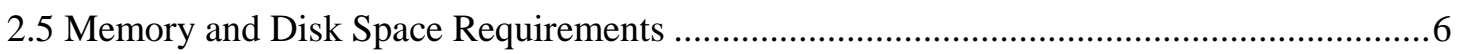

2.5.1 DESCARTES Memory and Disk Space Requirements ......................................6

2.5.2 CiderF Memory and Disk Space Requirements ..................................................

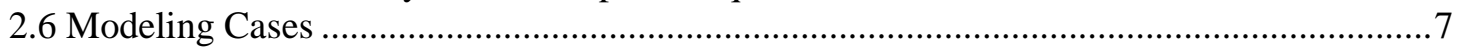

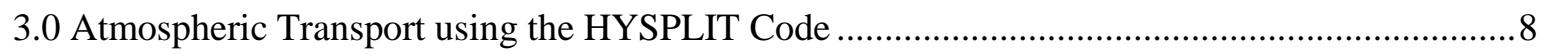

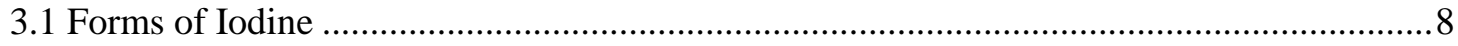

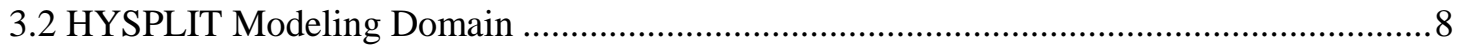

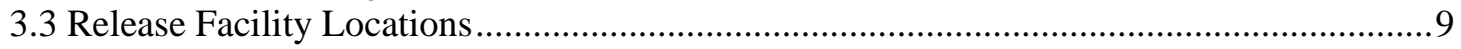

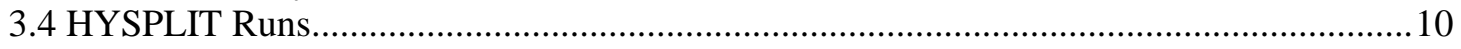

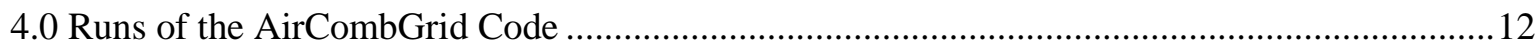

4.1 AirGrid Utility Code................................................................................................. 12

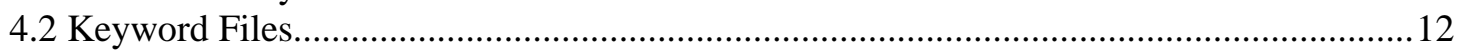

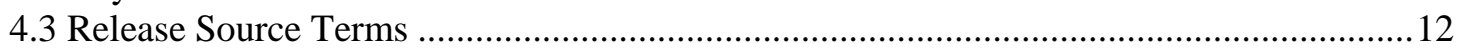

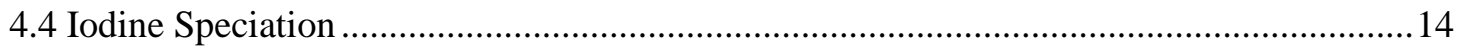

4.5 Example Results from the AirCombGrid Code..............................................................15

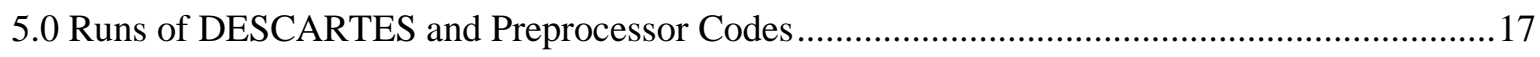

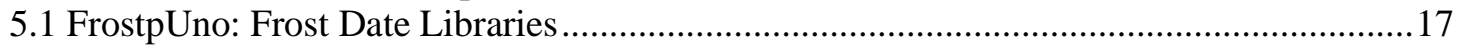

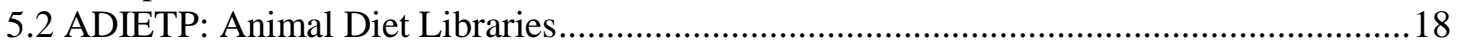

5.3 RECIPE: Commercial Leafy Vegetables.................................................................19

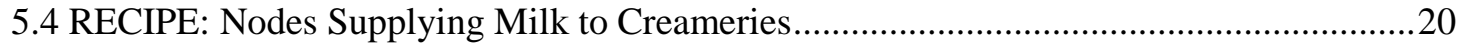

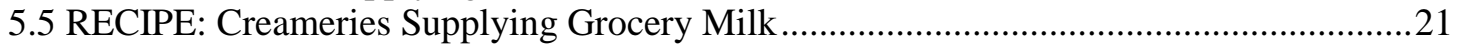

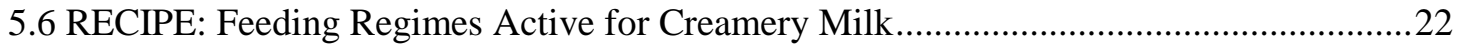

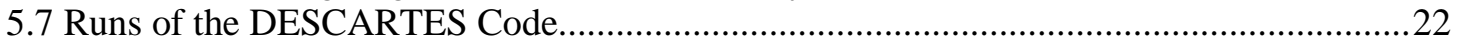

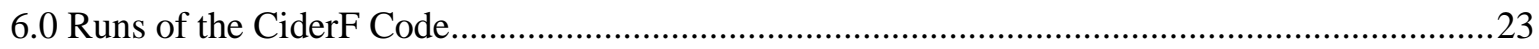

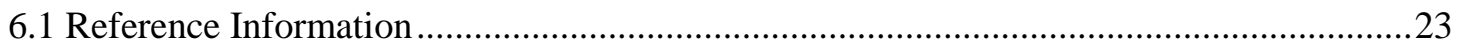

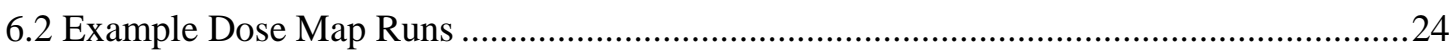

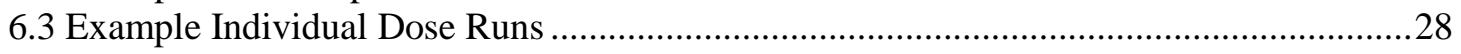

7.0 Data Package

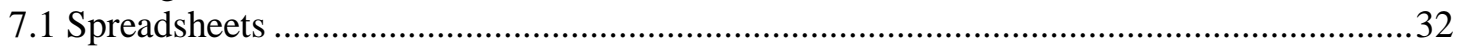

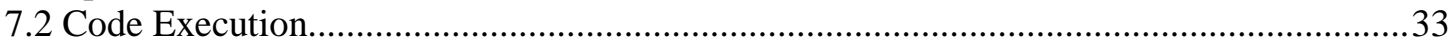

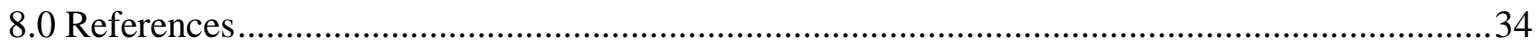




\section{Tables}

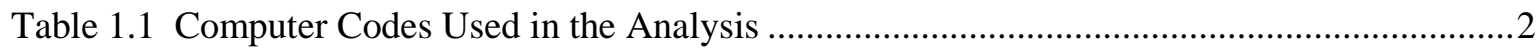

Table 3.1 Facility Locations for Release Modeling...................................................................9

Table 3.2 Runs of the srcSetupRunsMayak code (HYSPLIT run preparation) ...............................10

Table 3.3 Example Keyword File for the srcSetupRuns Code ..................................................11

Table 4.1 Runs of the AirCombGrid Code ..................................................................................12

Table 4.2 Summary Statistics on Total Release (Ci) by Facility for a 250 Realization Run ...........14

Table 4.3 Summary Statistics on Iodine Speciation Fractions Based on 250 Realizations .............15

Table 5.1 Keyword Files and Runs for the FrostpUno Code ......................................................17

Table 5.2 Keyword Files and Runs for the ADIETP Code....................................................19

Table 5.3 Keyword Files for the RECIPE code for Commercial Leafy Vegetables.......................19

Table 5.4 Keyword Files for the RECIPE code for Creamery Milk Production ............................20

Table 5.5 Keyword Files for the RECIPE code for Grocery Milk Recipes ...................................21

Table 5.6 Keyword Files and Runs for the RECIPE Code .........................................................22

Table 5.7 Keyword Files and Runs for the DESCARTES Code .................................................22

Table 6.1 Reference Diet, Lifestyle and Exposure Factors Keyword Files for CiderF Code...........24

Table 6.2 Reference Diet Definitions by Lifestyle Categories ....................................................24

Table 6.3 Keyword Files for the Best Estimate Map Run of the CiderF Code...............................25

Table 6.4 Keyword Files for the Stochastic Map Run for a Rural Child......................................26

Table 6.5 Keyword Files for the Stochastic Map Run for a Worker .............................................26

Table 6.6 Keyword Files for Map Cases Using the CiderF Code................................................29

Table 6.7 Percent of Variability in Thyroid Dose to a 5 Year Old Child Living in Ozersk in 1950

Attributable to Variability in Facility Releases and Atmospheric Transport............................29

\section{Figures}

Figure 2.1 Computational Grid for the Dose Model ...............................................................

Figure 3.1 Facility Locations for Release Modeling...............................................................10

Figure 4.1 Monthly Best Estimate Atmospheric Releases of ${ }^{131}$ I by Facility ..................................13

Figure 4.2 Annual Best Estimate Atmospheric Releases of ${ }^{131} \mathrm{I}$..................................................14

Figure 4.3 Example best estimate results from the AirCombGrid code for December 1948.

Monthly average air concentration $\left(\mathrm{Bq} / \mathrm{m}^{3}\right)$ (top pane) and month-end total decay corrected

deposition $\left(\mathrm{Bq} / \mathrm{m}^{2}\right)$ (bottom pane)................................................................................... 16

Figure 5.1 Historical temperature data for Balandino Airport, Chelyabinsk, Russian Federation ...18

Figure 6.1 Best Estimate Thyroid Dose (rad) for a Rural 5-Year-Old Child in 1949....................25

Figure 6.2 Stochastic Thyroid Dose (rad) for a Rural 5 Year Old Child in 1949 (1\% level in top pane, 99\% level in bottom pane)......................................................................................2

Figure 6.3 Median Thyroid Dose (Gy) for a Local Worker Due to Inhalation Totaled over the

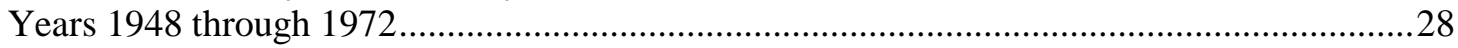

Figure 6.4 Annual Thyroid Dose for a 5 Year Old Male Child Living in Ozersk .........................30

Figure 6.5 Scatterplot of Thyroid Dose to a 5 Year Old Child Living in Ozersk in 1949 against the Internal Dose Conversion Factor ........................................................................................ 31 


\subsection{Introduction}

\subsection{Purpose}

This computational activity was conducted to produce representative dose estimates for members of the public living near Mayak, Russia, from atmospheric releases of ${ }^{131}$ I from the Mayak Production Association. This activity is part of a multi-year dose reconstruction effort (Mokrov et al. 2004). This document outlines the approach, code runs and data sets needed to calculate doses for members of the public living in or near Ozersk, Russia. It does not produce a complete suite of dose estimates or interpret the doses.

The approach to calculating individual doses to members of the public from historical releases of airborne ${ }^{131}$ I has the following general steps:

- Construct estimates of releases of ${ }^{131}$ I to the air from production facilities.

- Model the transport of ${ }^{131} \mathrm{I}$ in the air and subsequent deposition on the ground and vegetation.

- Model the accumulation of ${ }^{131} \mathrm{I}$ in soil, water and food products (environmental media).

- Calculate the dose for an individual by matching the appropriate lifestyle and consumption data for the individual to the concentrations of ${ }^{131} \mathrm{I}$ in environmental media at their residence location.

The sequence of calculations uses release estimates from facilities and atmospheric transport modeling to calculate ${ }^{131}$ I concentrations in environmental media (air, soil, and food products) on a grid of locations. The model region (grid of locations) is large enough to include the production of agricultural products supplied to residents of Ozersk from the collective farms identified in Figure 1 of (Mokrov et al. 2007a).

Some milk and leafy vegetable products consumed by residents of Ozersk came from a production and distribution system (collective farms) rather than being produced at the location where an individual lived. Therefore, the following two steps are used:

- Leafy Vegetable Distribution. A commercial distribution system for leafy vegetables is defined. This allows a city resident to eat leafy vegetables produced at a different location (a collective farm, for example).

- Milk Distribution: Milk was produced at private farms, collective farms and also from backyard animals. A milk distribution system is defined where a collective farm produces milk (called a creamery in the user guide for the DESCARTES code) and then supplies that milk to city residents (called a grocery in the user guide for the DESCARTES code). This technique models the movement of milk products from farms to consumers.

Three suites of calculations are identified in this analysis log. The first suite uses best estimates of all input parameters (best estimate case). The second suite uses stochastic inputs in a Monte Carlo approach to provide uncertainty estimates in the doses (stochastic case). A third suite uses stochastic releases and stochastic atmospheric transport, but best estimates for all other parameters (mono case). 


\subsection{Data Availability}

The series of milestone reports produced by the U.S.-Russian Joint Coordinating Committee on Radiation Effects Research, Project 1.4, (Rovny et al. 2009; Mokrov et al. 2009; Anspaugh and Napier 2009; Mokrov et al. 2008a; Mokrov et al. 2008b; Napier et al. 2008; Mokrov et al. 2007a; Mokrov et al. 2007b; Mokrov and Beregich 2006; Mokrov et al. 2004; Drozhko and Khokhryakov 2003) generally contain the data needed to run the suite of codes. Some of the data in those reports reference data collected for the Hanford Dose Reconstruction Project (Snyder et al. 1994). We note the source of any auxiliary data in later sections.

The historical atmospheric data needed to run the RATCHET code (Ramsdell Jr. et al. 1994) to produce air concentrations and surface deposition of ${ }^{131}$ I were not available at the time of this calculation. Instead, this calculation uses the HYSPLIT code (Draxler and Hess 1998). Meteorological data suitable for use with HYSPLIT are available on a web server (GDAS 2012).

Many of the data files or spreadsheets referenced in this document are provided in an electronic data package. See Section 7.0 for an overview of the electronic data package contents.

\subsection{Summary of Computer Code Runs}

The computer codes used in this calculation are identified in Table 1.1. The codes in Table 1.1 with documentation by Miley or Draxler have been tested extensively. The other codes have not been subjected to a formal testing process. A 64 bit version of Windows is required for the codes executing on a Windows platform.

Table 1.1 Computer Codes Used in the Analysis

\begin{tabular}{lcccc}
\hline Code & Platform & Documentation & Version & Revision \\
\hline srcSetupRuns & Linux & (Eslinger and Napier 2013) & M.01.001 & 24 Apr 2003 \\
\hline QsubRuns & Linux & (Eslinger and Napier 2013) & 3.04 .001 & 3 Apr 2013 \\
\hline HYSPLIT & Linux & (Draxler et al. 2012) & Serial & February 2013 \\
\hline AirGrid & Linux & Eslinger and Napier 2013) & 1.00.004 & 16 Jul 2013 \\
\hline AirCombGrid & Linux & (Eslinger and Napier 2013) & 1.00 .002 & 20 Jun 2013 \\
\hline AirCombGridView & Windows & (Eslinger and Napier 2013) & 1.01 .003 & 24 Jun 2013 \\
\hline DESCARTES & Windows & (Miley et al. 1994) & 4.004 & 16 Jul 2013 \\
\hline ADIETP & Windows & (Miley et al. 1994) & 3.1.B & 29 Nov 2012 \\
\hline FrostpUno & Windows & (Eslinger and Napier 2013) & 4.0.1 & 16 Jul 2013 \\
\hline RECIPE & Windows & (Miley et al. 1994) & 3.1.B & 30 Nov 2012 \\
\hline CiderF & Windows & (Eslinger and Napier 2013) & 1.03.016 & 12 Jun 2013 \\
\hline CiderView & Windows & (Eslinger and Napier 2013) & 1.02.002 & 24 Jun 2013 \\
\hline
\end{tabular}

The following sequence of computer code runs is required to build a new concentration data set for the dose code and to generate doses to individuals. Detailed information supporting the code runs is provided in later sections of this document. Indented bullets in the following list identify runs of support codes that assist the code in the previous bullet: 
- Run the HYSPLIT code multiple times to produce air concentration and surface deposition values across a large domain.

- Run the srcSetupRuns code to set up the individual HYSPLIT input files

- Run the QsubRuns code to schedule execution of the all of the HYSPLIT runs using unit releases

- $\quad$ Run the AirGrid code to convert large HYSPLIT output files into small files containing concentrations and depositions only at the locations used in the dose codes

- Run the AirCombGrid code to combine multiple output files written by the AirGrid code into two files containing air concentration and deposition data that are scaled for historical facility releases

- Run the DESCARTES code to produce concentrations in plant product media; animal product media (beef, goats, eggs, and poultry); herd and individual cow milk media; creamery and grocery milk media; commercial leafy vegetable media, and surface soil.

- Run the FrostpUno code to generate frost date libraries for each modeled year

- Run the ADIETP program to generate animal diet libraries

- Run the FrostpUno code to generate feeding season date libraries

- Run the RECIPE program to generate the recipe for milk cow feeding regimes contributing to creamery milk at each node

- Run the RECIPE program to generate the recipe for which nodes supply milk to each creamery

- Run the RECIPE program to generate the recipe for which creameries supply milk to which grocery stores

- Run the RECIPE program to generate the recipe for which nodes supply commercial leafy vegetables to consumption nodes

- $\quad$ Run the CiderF code to produce dose estimates

- Run the CiderView code to produce maps in KML format for viewing in Google Earth (Google 2013) 


\subsection{Base Problem Definition}

Incorporation of the existing data into model inputs uses several base assumptions. The base assumptions are addressed in this section.

\subsection{Time Period}

Data on the monthly releases of ${ }^{131}$ I to the atmosphere are provided in (Mokrov et al. 2008a). Reactor A began operation in June, 1948, and the first processing runs at B plant occurred in December, 1948.

Tabulated data for monthly releases from the production stacks are provided from 1948 through 1967, and graphical results based on monitoring data are shown for later years. The releases in early years are much higher than in later years, with very small releases occurring after 1972. Therefore, this suite of calculations uses a time period beginning in June, 1948, and extending through December, 1972.

\subsection{Environmental Concentration Averaging Time}

The DESCARTES code can export the concentrations of ${ }^{131}$ I in air, soil and food products for use in the CiderF code on a daily, weekly or monthly basis. The anticipated use of the CiderF code is to calculate annual or lifetime dose and this quantity should not be highly sensitive to daily fluctuations in concentrations about an average monthly concentration. Therefore, this analysis uses monthly outputs from DESCARTES (averaged over all days in the month).

An auxiliary analysis, not otherwise documented, was conducted to examine the assumption of using monthly average concentrations. The annual dose to an individual living in Ozersk in 1948 calculated on daily outputs from DESCARTES differs from annual doses calculated from monthly outputs from DESCARTES by a relative amount of $0.2 \%$ or less.

\subsection{Dose Model Region}

The dose model region is local to Ozersk and is large enough to include the production of agricultural products potentially consumed by residents of Ozersk. Food production areas supplying agricultural products for the residents of Ozersk are provided in Figure 1 of (Mokrov et al. 2007a). To ensure the dose model locations are consistent across all of the computer codes, a grid of locations (a point on this grid is called a node in the code documentation) is adopted. This grid consists of a rectangular region around the production facilities and an irregularly shaped region that covers the Techa River from near the release facilities to its discharge into the Iset River. The rectangular portion of the grid (node numbers 1 through 516) has the following characteristics:

- North to south extent: North Latitude $55.1^{\circ}$ to $55.95^{\circ}$ in steps of $0.05^{\circ}$

- East to west extent: East longitude $60.0^{\circ}$ to $62.1^{\circ}$ in steps of $0.1^{\circ}$

A portion of the region near Ozersk is divided into a finer resolution grid:

- North to south extent: North Latitude $55.5^{\circ}$ to $55.8^{\circ}$ in steps of $0.025^{\circ}$

- East to west extent: East longitude $60.5^{\circ}$ to $61.1^{\circ}$ in steps of $0.05^{\circ}$

The resulting dose model grid contains 618 locations and is shown overlain on a local aerial view in Figure 2.1. The dose model domain covers a region about $16,000 \mathrm{~km}^{2}$ in size. 


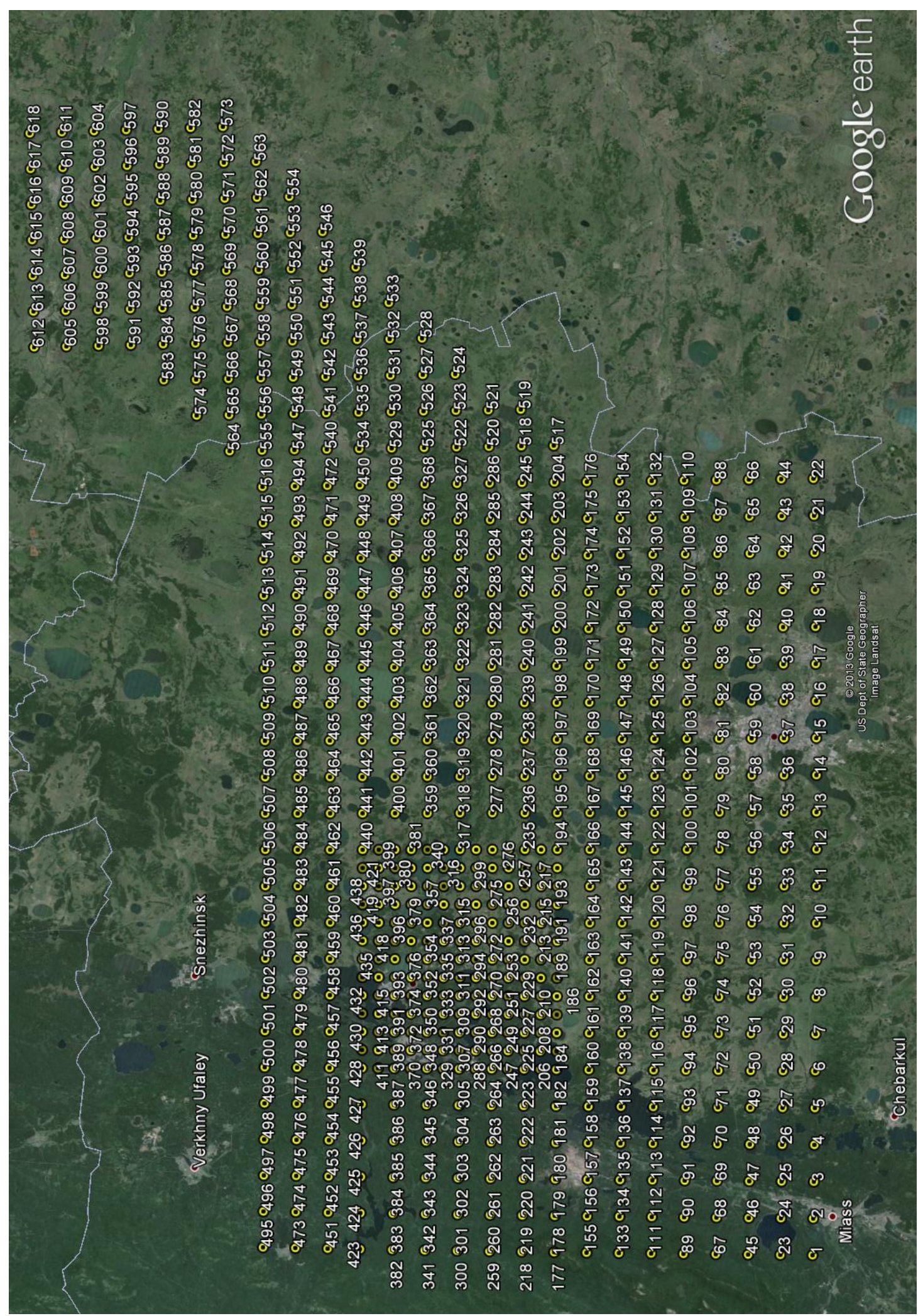

Figure 2.1 Computational Grid for the Dose Model 
The spreadsheet named Grid.xlsx contains the information needed to produce the NODE keywords used by the AirGrid, AirCombGrid, AirCombGridView and CiderView codes. The keywords are produced in the spreadsheet and they can be copied and pasted into the text input files for these codes. The AirCombGridView and CiderView codes produce contoured outputs in KML format that can be viewed using Google Earth (Google 2013). Two additional files are provided for the convenience of showing the node locations in Google Earth. These files are the following:

- MayakGridNumbered.kmz This zipped KML file can be opened directly with Google Earth. It contains icons for the 618 node locations that are labeled with the node numbers.

- MayakGrid.kmz This zipped KML file can be opened directly with Google Earth. It contains icons for the 618 node locations but it does not include the node numbers.

Figure 2.1 shows the dose model domain with the file MayakGridNumbered.kmz. This display can be useful for identifying the node number associated with the location where an individual lived.

\subsection{Commercial Foods}

Although the modeling domain is large enough to cover many of the towns around Ozersk, including Chelyabinsk, commercial food production information is available only for residents of Ozersk. Food production areas supplying agricultural products for the residents of Ozersk are provided in Figure 1 of (Mokrov et al. 2007a). In this suite of calculations, a creamery (see the DESCARTES user's guide (Miley et al. 1994) for the use of creameries) is synonymous with a collective farm.

Further information about commercial food production and distribution are provided in later sections. See section 5.3 for information about production of commercial leafy vegetables. See sections 5.4 and 5.5 for information about the production and distribution of milk.

\subsection{Memory and Disk Space Requirements}

Example memory and disk space usage for the DESCARTES and CiderF codes are provided in the following sections.

\subsubsection{DESCARTES Memory and Disk Space Requirements}

The DESCARTES code performs dynamic memory allocation, so different runs will use different amounts of memory. A run using 1 realization on a domain of 618 nodes utilized $7 \mathrm{Mb}$ of memory. A run of 250 realizations on a domain of 618 nodes used $225 \mathrm{Mb}$ of memory.

A stochastic run of DESCARTES using 250 realizations on 618 nodes for the years 1948 through 1972 requires about $10.3 \mathrm{~Gb}$ of disk space for input files. In addition, the output files occupy another $4 \mathrm{~Gb}$ of disk space.

A determinist run of DESCARTES (1 realization) on 618 nodes for the years 1948 through 1972 requires about $169 \mathrm{Mb}$ of disk space for input files. In addition, the output files occupy another $96.2 \mathrm{Mb}$ of disk space. 


\subsubsection{CiderF Memory and Disk Space Requirements}

The CiderF code performs dynamic memory allocation, so different runs will use different amounts of memory. For example, a run of individual cases using 1 realization on the time period 1948 through 1972 utilized $36 \mathrm{Mb}$ of memory. In contrast, a map run of 250 realizations for a domain with 618 nodes utilized $342 \mathrm{Mb}$ of memory when only annual maps are output. A map run that totals doses on the time period 1948 through 1972 utilized 781 Mb of memory.

The CiderF code can be executed multiple times after the air transport and environmental accumulation codes have been run without rerunning the previous codes. A number of data files output by the DESCARTES code are used as input to CiderF. The disk space requirements for these data files depend on the options selected in DESCARTES. Disk space requirements for several cases using 618 nodes on the time period 1948 through 1972 are as follows:

- A single realization case requires about $96 \mathrm{Mb}$ of disk space for CiderF input files.

- A single realization case requires about $265 \mathrm{Mb}$ of disk space for input files for both DESCARTES and CiderF.

- A 250 realization case requires about $4 \mathrm{~Gb}$ of disk space for CiderF input files.

- A 250 realization case requires about $14.3 \mathrm{~Gb}$ of disk space for input files for both DESCARTES and CiderF.

\subsection{Modeling Cases}

Results identified in this document are calculated for both deterministic and stochastic runs. The runs are grouped in the following fashion:

- Best Estimate Case: Deterministic runs (one realization) are performed using "best estimates" for each input parameter. The "best estimates" are chosen as representative (mean, median or single value based on expert judgment) values rather than bounding values. The atmospheric transport model uses an average $\chi / Q$ field.

- Stochastic Case: The stochastic runs are based on the available data ranges or statistical definitions of a large number of input variables. Specifics of the stochastic definitions will be supplied in later document sections. 


\subsection{Atmospheric Transport using the HYSPLIT Code}

We do not have access to detailed meteorological data for the Mayak region in 1948 through 1972, however, detailed meteorological data since 2007 are available (GDAS 2012). Therefore, a two-step approach is adopted. First, the parallel version (February 2013) of the HYSPLIT computer code for Linux is used to produce time sequences of ${ }^{131}$ I air concentration and deposition assuming unit releases on a daily basis using meteorological data from 2007 through 2011. Second, the historical release ${ }^{131}$ I rates from each facility are used to combine the unit release results into time sequences of surface deposition and air concentrations for 1948 through 1972.

These results are representative of air movement around Mayak during the different months of the year, but they will not exactly match with "real" air movement in any specific year in the dose modeling time period. The user instructions for the AirCombGrid code (section 6 of (Eslinger and Napier 2013)) contains further information on this technique. In summary, $\chi / \mathrm{Q}$ values for the years 2007 through 2011 are applied to the years 1948 through 1972.

\subsection{Forms of lodine}

The released ${ }^{131}$ I can propagate in organic, elemental or particulate forms (see section 4.4 for more information). The HYSPLIT code can model all of these modes, but not the interchange between the forms. Thus, each HYSPLIT run used a unit source of all three forms. The air concentration and ground deposition of each form were tracked separately. The parameters governing the deposition rates are an input to HYSPLIT. Although the specific values of the deposition rates may have a significant impact on the environmental concentrations, implementing them in a fully stochastic manner requires making a prohibitive number of HYSPLIT runs. Therefore, to limit the computational burden, fixed nominal values are used for these HYSPLIT parameters. The nominal values are as follows:

- Organic (Orgn): Model organic iodine as a gas, with no wet deposition and no dry deposition.

- $\quad$ Elemental (Elem): Model elemental iodine as a depositing gas.

o Settling velocity $=10 \mathrm{~cm} / \mathrm{s}$

- Particulates (Part): Model particulate iodine, where the deposition is affected by both wet and dry mechanisms.

o Settling velocity $=1 \mathrm{~cm} / \mathrm{s}$

o Wet deposition in-cloud $=4 \mathrm{E} 4$ (g Iodine $/ \mathrm{L}$ air to g Iodine $/ \mathrm{L}$ in rain at surface)

o Wet removal below cloud $=5 \mathrm{E}-5(1 / \mathrm{s})$ time removal constant

\subsection{HYSPLIT Modeling Domain}

The HYSPLIT model computational grid is centered at north latitude $55.5^{\circ}$ and east longitude $61.0^{\circ}$ with a span of $4^{\circ}$ in latitude and $8^{\circ}$ in longitude using $0.025^{\circ}$ increments in both latitude and longitude. The air transport domain is much larger than the dose domain to account for movement of air across the domain of interest.

Although air patterns can sometimes cause a released plume to return to the local region days after it is released, the concentration levels in such plumes are typically much lower than in the original plume. 
Therefore, for purposes of computational efficiency, individual plumes are only tracked for 96 hours after they are released.

\subsection{Release Facility Locations}

The different reactors and radiochemical facilities operated on different time periods. We model daily releases from the facilities and then use a post processing step to overlay scaled individual releases into appropriate time sequences (1948 through 1972). Unit source terms are modeled from the following facilities: A, AI, AV1, AV2, AV3, OK (combined) reactors, and radiochemical plants B and DB. The geographic locations of the facilities and the height of the release stacks are provided in Table 3.1. These release points are also captured in the spreadsheet named Release_Points.xlsx. A snapshot of a Google Earth (Google 2013) image showing the facility locations is provided in Figure 3.1.

Table 3.1 Facility Locations for Release Modeling

\begin{tabular}{lllll}
\hline ID & $\begin{array}{l}\text { Latitude } \\
(\mathrm{deg})\end{array}$ & $\begin{array}{l}\text { Longitude } \\
(\mathrm{deg})\end{array}$ & $\begin{array}{l}\text { Stack Height } \\
(\mathrm{m})\end{array}$ & Description \\
\hline A & 55.70194444 & 60.78305556 & 95 & A Reactor \\
\hline AI & 55.70166667 & 60.78055556 & 95 & AI Reactor \\
\hline AV3 & 55.70388889 & 60.78277778 & 95 & AV3 Reactor \\
\hline AV1 & 55.705 & 60.81138889 & 80 & AV1 Reactor \\
\hline AV2 & 55.70666667 & 60.80305556 & 80 & AV2 Reactor \\
\hline OK & 55.69888889 & 60.7825 & 80 & OK Reactor Complex \\
\hline B & 55.68805556 & 60.79888889 & 150 & B Plant Stacks \\
\hline DB & 55.69722222 & 60.80416667 & 150 & DB Plant Stacks \\
\hline
\end{tabular}

For viewing convenience, the information in Table 3.1 is also provided in a file named MayakFacilities.kml. This file can be opened directly in Google Earth. 


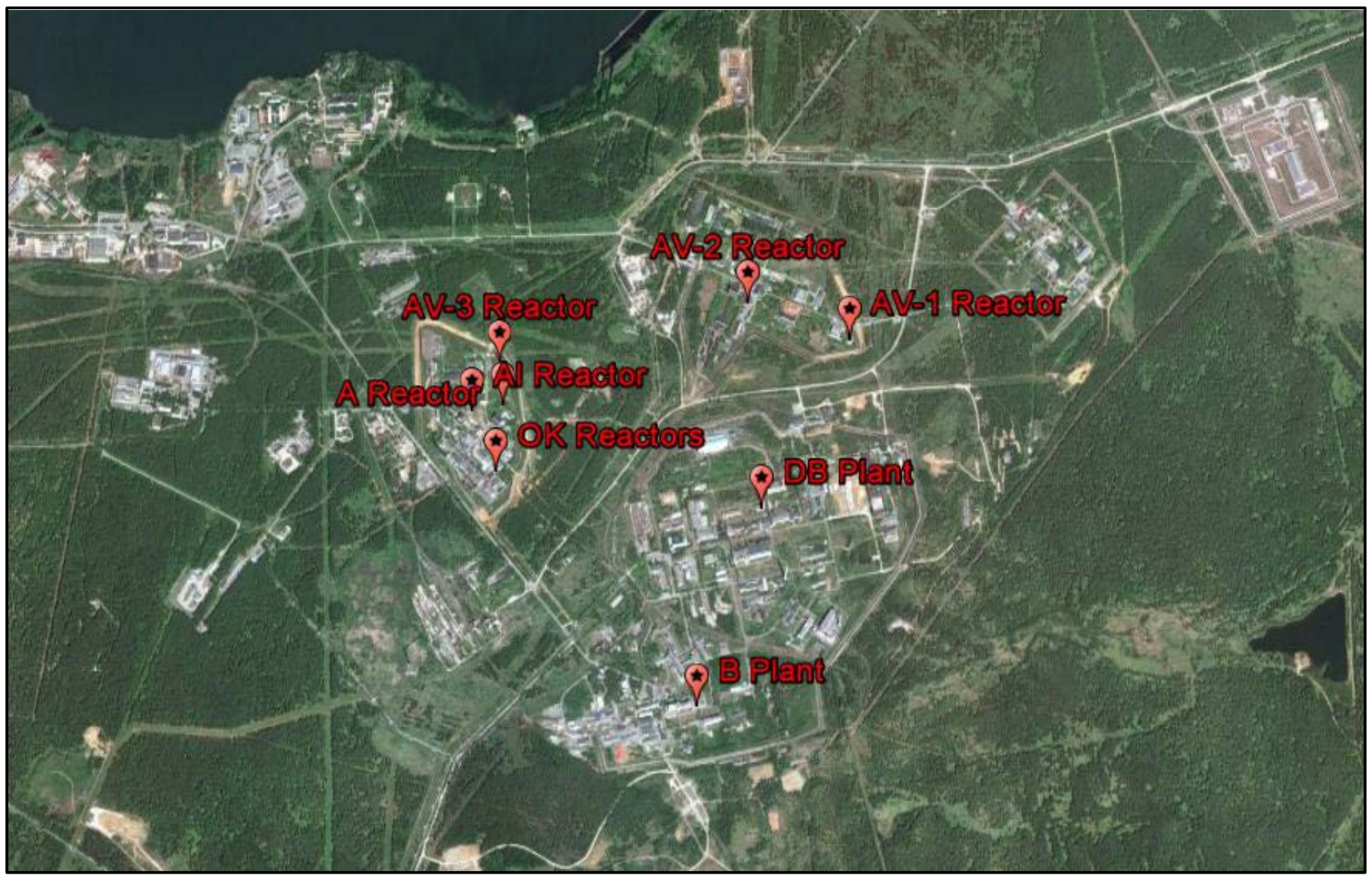

Figure 3.1 Facility Locations for Release Modeling

\subsection{HYSPLIT Runs}

Setting up and executing the HYSPLIT runs has the following general steps. First, a keyword file is prepared for the srcSetupRunsMayak code. In the user's guide (Eslinger and Napier 2013), this code is simply called srcSetupRuns. Execution of the code creates run directories and prepares control files for HYSPLIT. Then, the QsubRuns code uses files prepared by the srcSetupRuns code to submit individual HYSPLIT jobs to a batch queue. Each of three runs of the srcSetupRuns code uses a slightly different keyword file. The modifications are the release stack height and the list of facilities to model. The three runs of the srcSetupRuns code are identified in Table 3.2. A total of 14,632 runs of the HYSPLIT code were required to model 8 release locations every day for just over five years. Each HYSPLIT run was followed by a run of a utility program that converted the binary HYSPLIT output file to ASCII format.

Table 3.2 Runs of the srcSetupRunsMayak code (HYSPLIT run preparation)

\begin{tabular}{lcll}
\hline Purpose & Stack Height $(\mathrm{m})$ & Keyword File & Station List File \\
\hline Reactors: AV1, AV2, OK & 80 & Mayak.kwd & Station_List_Reactor80.txt \\
\hline Reactors: A, AI, AV3 & 95 & Mayak.kwd & Station_List_Reactor95.txt \\
\hline Radiochemical plants: B, DB & 150 & Mayak.kwd & Station_List_Plants150.txt \\
\hline
\end{tabular}

The HYSPLIT code can be run in puff or particle modes. The 3-D particle mode for both vertical and horizontal air movement is used in all of the HYSPLIT runs. Each run used 250,000 particles. 
The release facilities are located within a region about $2 \mathrm{~km}$ across and this is a very small region when considering atmospheric transport for hundreds of km. However, a few preliminary runs of the air transport model indicated that modeled air concentrations in Ozersk were sensitive to both the release height and the release point location. Therefore, atmospheric transport was modeled for each facility separately rather than combining the facilities into groups with the same release height.

An example keyword file for the srcSetupRunsMayak code is provided in Table 3.3.

Table 3.3 Example Keyword File for the srcSetupRuns Code

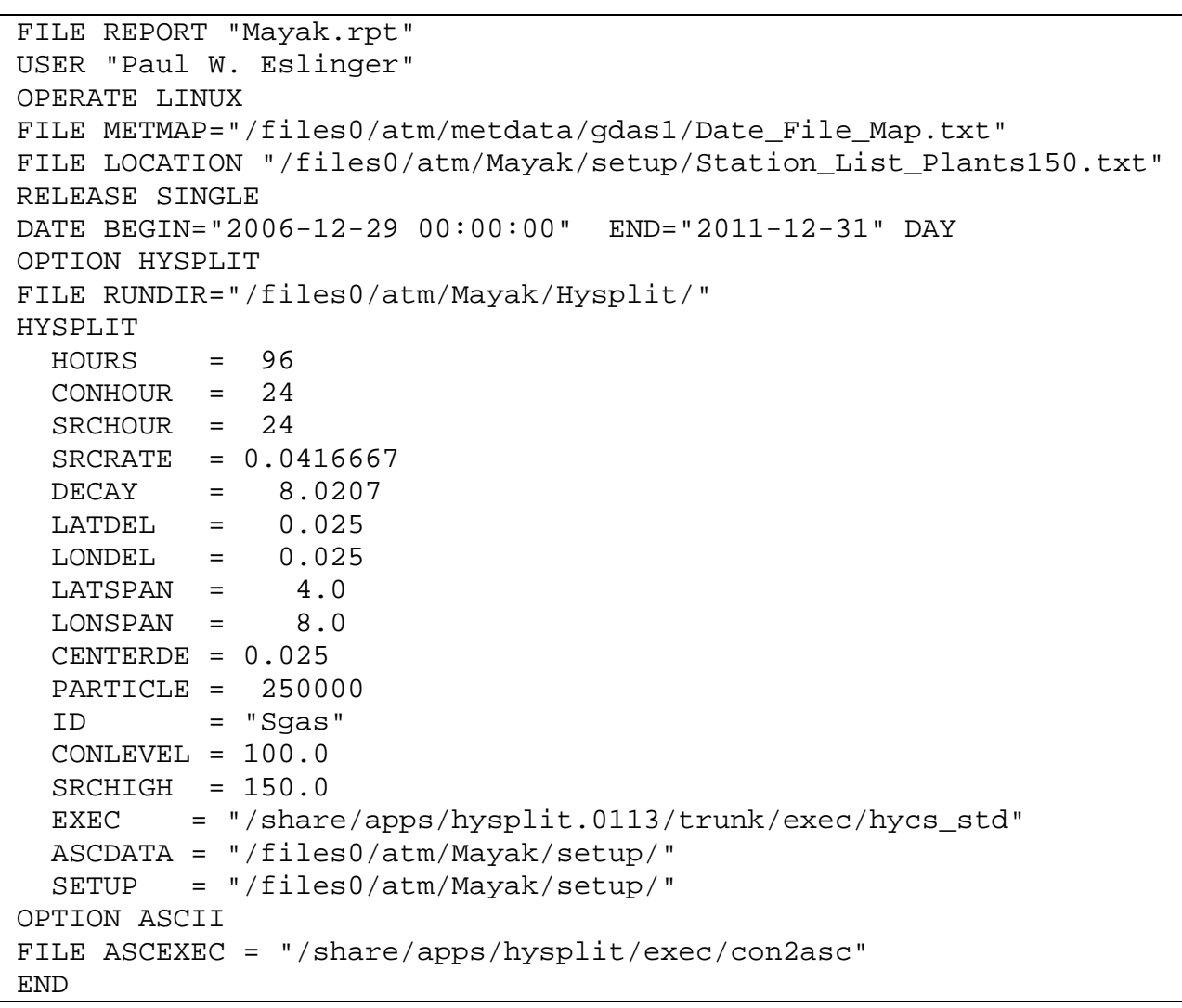




\subsection{Runs of the AirCombGrid Code}

The reactors and radiochemical facilities operated at different time periods. The AirCombGrid code applies individual releases from facilities (from 1948 through 1972) onto the results of the air transport modeling performed with unit release assumptions. The AirCombGrid code writes air concentration and air deposition files in the correct format for use in the DESCARTES code.

\subsection{AirGrid Utility Code}

The AirGrid code is used to register the concentrations and depositions produced by the HYSPLIT code on the set of locations (nodes) to be used in the AirCombGrid, DESCARTES and CiderF codes. This intermediate step greatly reduces the amount of $\mathrm{I} / \mathrm{O}$ needed in the AirCombGrid code relative to reading HYSPLIT produced files directly.

The AirGrid code was used with the input file named AirGrid.kwd. This code executed on the Linux cluster where the HYSPLIT code was used. The AriGrid run finished in about 6 hours.

\subsection{Keyword Files}

The two runs of the AirCombGrid code are identified in Table 4.1. The best estimate case ran in about 3 hours. The stochastic case ran in about 3.5 hours.

Table 4.1 Runs of the AirCombGrid Code

\begin{tabular}{ll}
\hline Purpose & Keyword File \\
\hline Best Estimate Case & Best.kwd \\
\hline Stochastic Case & Stochastic.kwd \\
\hline
\end{tabular}

\subsection{Release Source Terms}

Monthly releases of ${ }^{131}$ I are available (Mokrov et al. 2008a), on pages 23-24 for the D and DB radiochemical plants. Annual releases from the reactors are provided in Table 4.1 of the same report. Some release data for 1968 through 1972 can be derived from the stack monitoring values of the same report. The typical use of CiderF is to calculate annual or lifetime doses, so using monthly source terms from the largest releases should not cause a significant loss of information.

Deterministic release estimates use the best estimate monthly values, implemented as constant daily releases for each day in the month. If published monthly values are not available, then the annual values are divided into equal releases for each day. Stochastic releases are implemented as a scaling factor (triangular distribution on [minimum, best estimate, maximum]) for the annual releases.

The keyword files identified in Table 4.1contain source entries for every month a facility operated. Preparation of the release keywords is performed in a spreadsheet and then the keywords are copied and pasted into the text keyword files. The spreadsheet is named Release_Keywords.xlsx and it contains a 
separate sheet for every release facility. Stochastic keywords are located in column S of each facility sheet and best estimate keywords are located in column $\mathrm{U}$ of each facility sheet.

The AirCombGrid code generates monthly and annual releases from every facility. The monthly releases for each realization are scaled to match the annual release for that realization.

Best estimate values for the monthly releases (Ci) by facility, as output by the AirCombGrid code, are provided in Figure 4.1. As expected, releases from the radiochemical processing plants B and DB dominate the releases. The best estimate annual releases for all active facilities, as output by the AirCombGrid code, are provided in Figure 4.2.

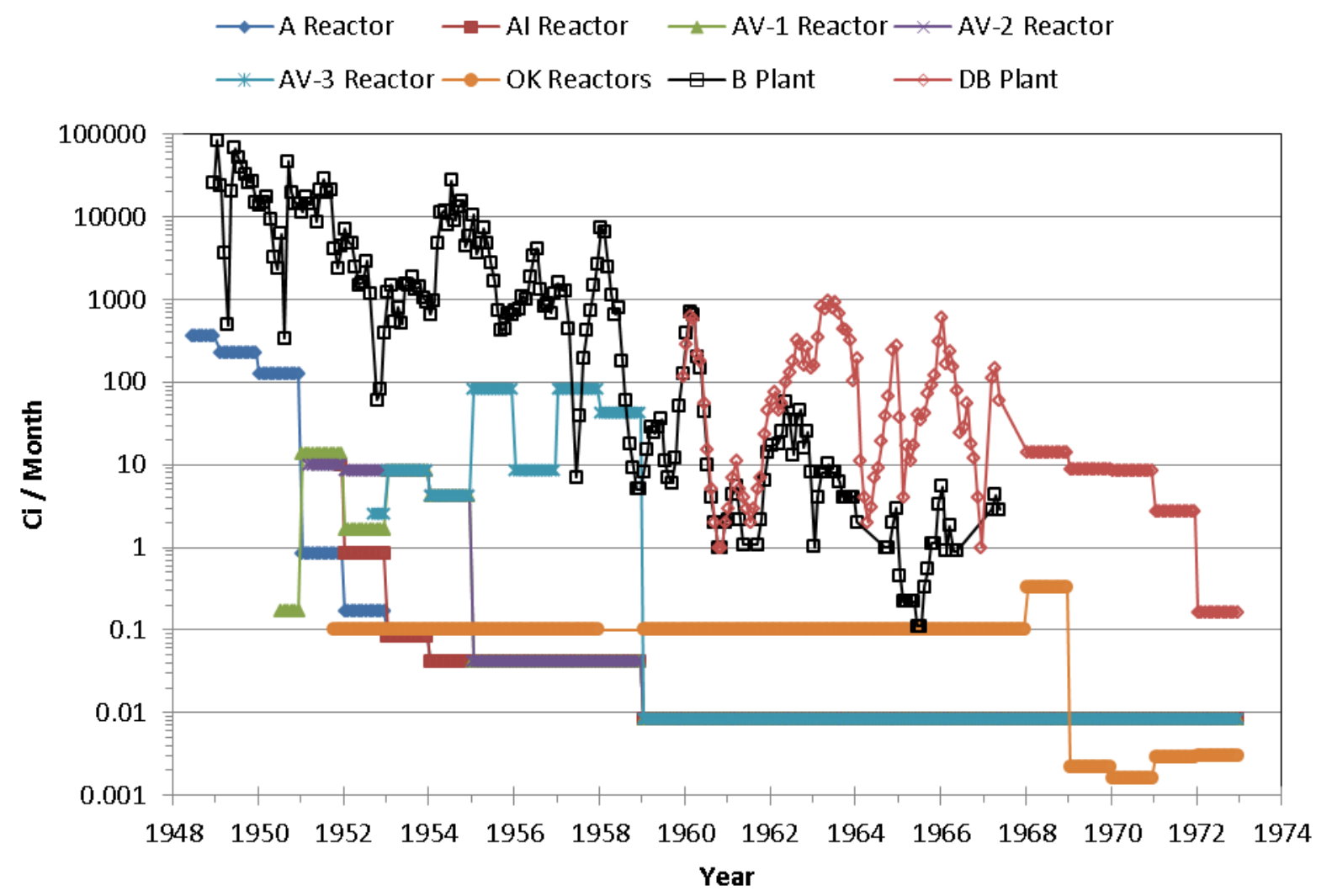

Figure 4.1 Monthly Best Estimate Atmospheric Releases of ${ }^{131}$ I by Facility 


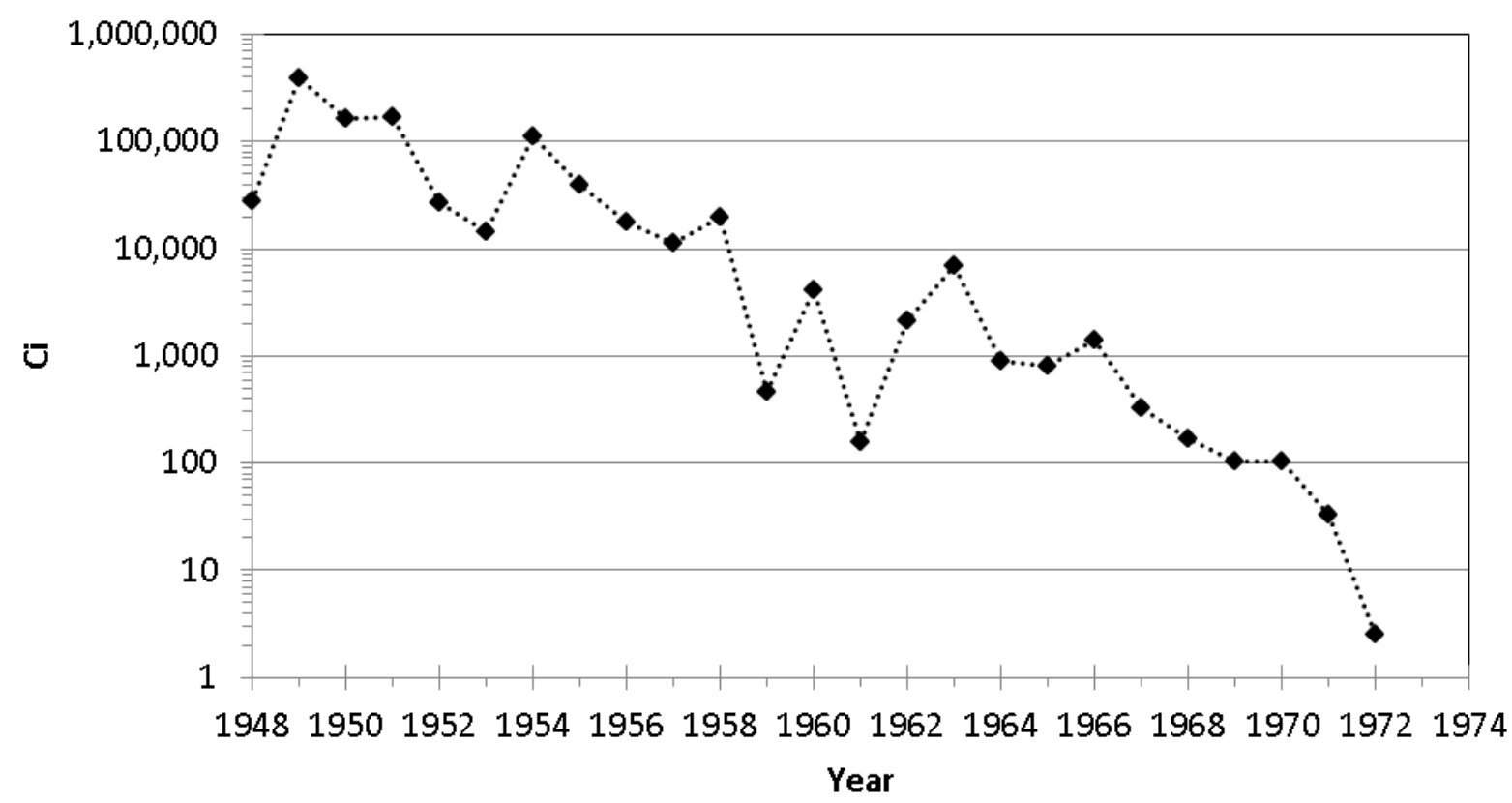

Figure 4.2 Annual Best Estimate Atmospheric Releases of ${ }^{131} \mathrm{I}$

Summary statistics on the total release of ${ }^{131} \mathrm{I}$ (Ci) to the air by facility for the years 1948 through 1972 are provided in Table 4.2. The summary was developed from the 250 realization stochastic case.

Table 4.2 Summary Statistics on Total Release (Ci) by Facility for a 250 Realization Run

\begin{tabular}{lllllll}
\hline \multirow{2}{*}{ Facility } & Minimum & $\begin{array}{c}2^{\text {th }} \\
\text { Percentile }\end{array}$ & Median & $\begin{array}{c}7^{\text {th }} \\
\text { Percentile }\end{array}$ & Maximum & $\begin{array}{c}\text { Standard } \\
\text { Deviation }\end{array}$ \\
\hline A & 5,432 & 6,173 & 6,503 & 6,778 & 7,620 & 437.4 \\
\hline AI & 20.39 & 23.67 & 24.74 & 25.73 & 29.91 & 1.565 \\
\hline AV1 & 275.1 & 316.7 & 334.1 & 345.9 & 385.0 & 21.03 \\
\hline AV2 & 285.3 & 335.2 & 351.3 & 367.1 & 400.1 & 21.17 \\
\hline AV3 & 2,294 & 2,613 & 2,751 & 2,866 & 3,228 & 180.6 \\
\hline B & 844,300 & 940,000 & 982,500 & $1,022,900$ & $1,141,900$ & 56,790 \\
\hline DB & 12,310 & 13,900 & 14,520 & 15,080 & 16,890 & 840.1 \\
\hline OK & 20.29 & 21.74 & 22.17 & 22.59 & 24.22 & 0.6507 \\
\hline
\end{tabular}

\section{4 lodine Speciation}

The speciation fraction algorithm for ${ }^{131}$ I embedded in the AirCombGrid code is taken from (Napier et al. 2008). The algorithm has two steps. First, the fraction of ${ }^{131} \mathrm{I}$ in each form is sampled uniformly from within its specific range. Second, the three fractions are normalized so the total equals one. The fractions of each form are defined as follows:

- elemental iodine, uniformly distributed on $(0.10,0.45)$,

- organic iodine, uniformly distributed on $(0.20,0.35)$, and

- $\quad$ particulate iodine, uniformly distributed on $(0.20,0.65)$. 
Summary statistics on the iodine speciation fractions from the 250 realization run of the AirCombGrid are provided in Table 4.3. For any single realization, the speciation fractions of the three iodine types sum to one. However, the summary statistics in Table 4.3 are provided for each iodine type individually over the set of 250 values. The statistics (percentiles, standard deviation, mean, etc.) only sum to one over the three iodine types for the mean. Speciation fractions for the best estimate run uses 0.44 for particles, 0.29 for the organic form and 0.27 for the elemental form. These values are approximately the mean values from the stochastic set of 250 speciation fractions.

Table 4.3 Summary Statistics on Iodine Speciation Fractions Based on 250 Realizations

\begin{tabular}{lrrr}
\hline Statistic & Particulate & Organic & Elemental \\
\hline Minimum & 0.2287 & 0.1696 & 0.1024 \\
\hline 1\% Level & 0.2337 & 0.1723 & 0.1126 \\
\hline 5\% Level & 0.2927 & 0.2045 & 0.1307 \\
\hline 10\% Level & 0.3108 & 0.2188 & 0.1472 \\
\hline 25\% Level & 0.3689 & 0.2457 & 0.1902 \\
\hline Median & 0.4414 & 0.2850 & 0.2576 \\
\hline 75\% Level & 0.5078 & 0.3343 & 0.3361 \\
\hline 90\% Level & 0.5610 & 0.3896 & 0.3901 \\
\hline 95\% Level & 0.5875 & 0.4130 & 0.4233 \\
\hline 99\% Level & 0.6391 & 0.4480 & 0.4726 \\
\hline Maximum & 0.6433 & 0.5040 & 0.4986 \\
\hline Mean & 0.4391 & 0.2953 & 0.2656 \\
\hline St. Dev. & 0.0932 & 0.0644 & 0.0915 \\
\hline
\end{tabular}

\subsection{Example Results from the AirCombGrid Code}

Example results from the AirCombGrid code (displayed as interpolated contours) are provided in Figure 4.3 for December 1948 for the best estimate case. The top pane shows the average air concentration over the month and the bottom pane shows the month-end decay corrected total deposition. The activity units in these plots are $\mathrm{Bq}$, rather than $\mathrm{Ci}$ used elsewhere in this document. The plots were produced using the AirCombGridView code and the keyword files named conc.kwd and depo.kwd. These files are located in the directory Mayak\Best\AirCombGrid in the electronic data package. 

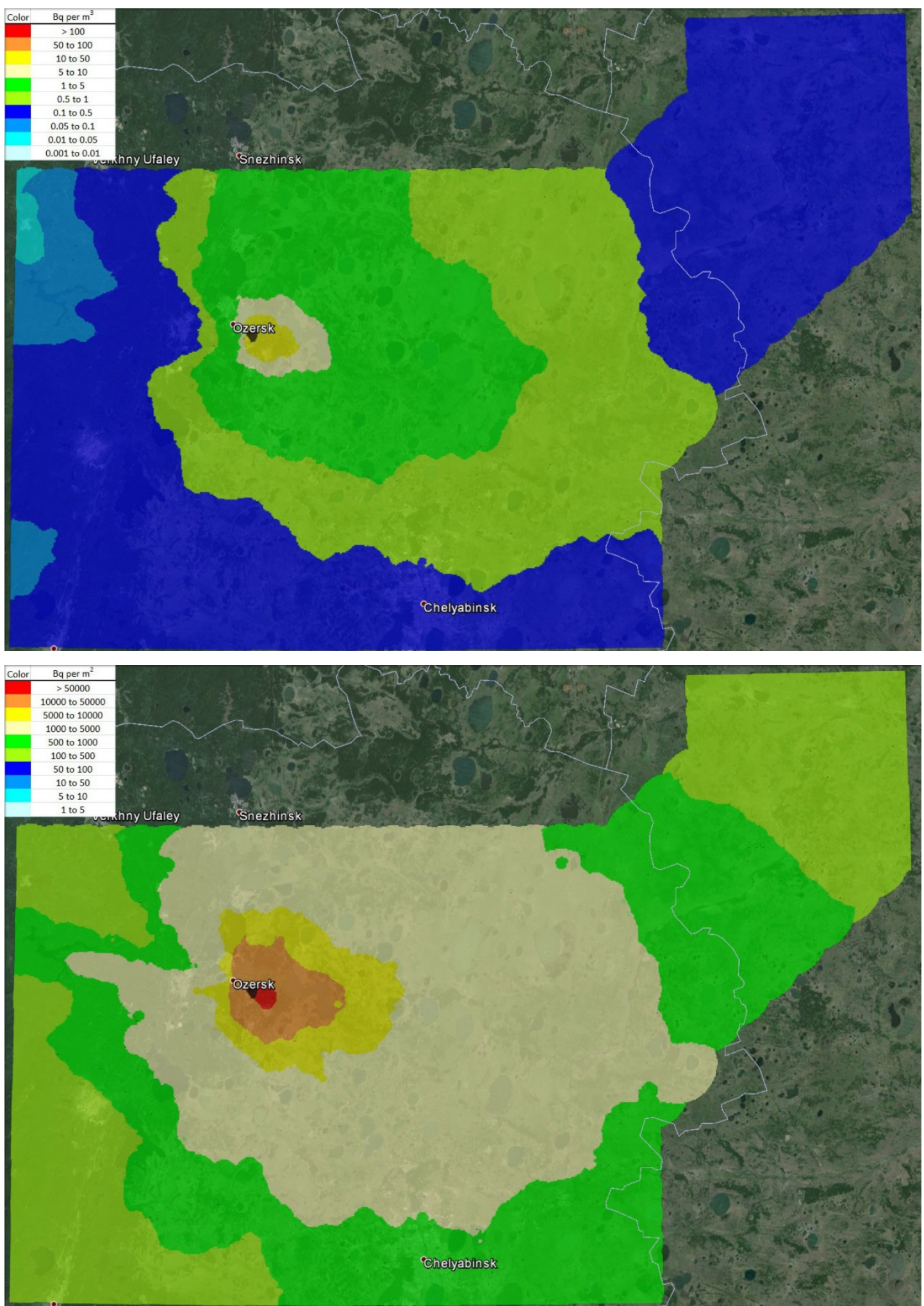

Figure 4.3 Example best estimate results from the AirCombGrid code for December 1948. Monthly average air concentration $\left(\mathrm{Bq} / \mathrm{m}^{3}\right)$ (top pane) and month-end total decay corrected deposition $\left(\mathrm{Bq} / \mathrm{m}^{2}\right)$ (bottom pane). 


\subsection{Runs of DESCARTES and Preprocessor Codes}

A suite of preprocessors is used to prepare data sets for the DESCARTES code for best estimate or stochastic data sets. The following three major cases were run in DESCARTES:

- Best Estimate Case: All DESCARTES parameters were set to best estimate values and the best estimate case from the AirCombGrid code is used (1 realization).

- Stochastic Case: A full set of stochastic inputs (250 realizations) is used in DESCARTES and the stochastic case from the AirCombGrid code.

- Mono Case: All DESCARTES parameters were set to best estimate values and the stochastic case from the AirCombGrid code is used. This 250 realization case is used to determine the variability in doses solely due to stochastic releases and stochastic air transport.

\subsection{FrostpUno: Frost Date Libraries}

A separate library file containing frost dates is required for each model year when computing plantrelated concentrations. These files are produced by the utility code FrostpUno. FrostpUno is a modification of the FROSTP code (Miley et al. 1994) that applies the same definition of frost dates for all locations in the dose model domain and multiple years. The code writes a file of randomized frost dates for every model year. The code runs are identified in Table 5.1.

Table 5.1 Keyword Files and Runs for the FrostpUno Code

\begin{tabular}{ll}
\hline Purpose & Keyword File \\
\hline Best Estimate Case & FrostBest.kwd \\
\hline Stochastic Case & FrostStoc.kwd \\
\hline Mono Case & FrostMono.kwd \\
\hline
\end{tabular}

Historical weather information for Baladino Airport, Chelyabinsk, Russian Federation is available on the web (WeatherSpark 2012). Reading from the interactive graph, reproduced here as static Figure 5.1, for averages on the "dashboard" at that website, one can obtain the following range of dates. Last spring frost (overnight low reached down to $32{ }^{\circ} \mathrm{F}$ ): average day is April 12 (Julian day 102); $10 \%$ are as early as March 30 (Julian day 89); 10\% are as late as May 2 (Julian day 122). First fall frost (overnight low reached down to $32{ }^{\circ} \mathrm{F}$ ): average day is October 24 (Julian day 297); $10 \%$ are as early as October 2 (Julian day 275); 10\% are as late as November 10 (Julian day 314).

The best estimate run uses a spring frost date of April 12 (Julian day 102) and a fall frost date of October 24 (Julian day 297). These dates are applied to all dose model locations. The same dates are used for every year.

The stochastic runs use uniform distributions for the frost dates. The spring date is uniform on the range $(89,122)$ and the fall date is uniform on the range $(275,314)$. These same stochastic definitions are used at all locations for every year. 


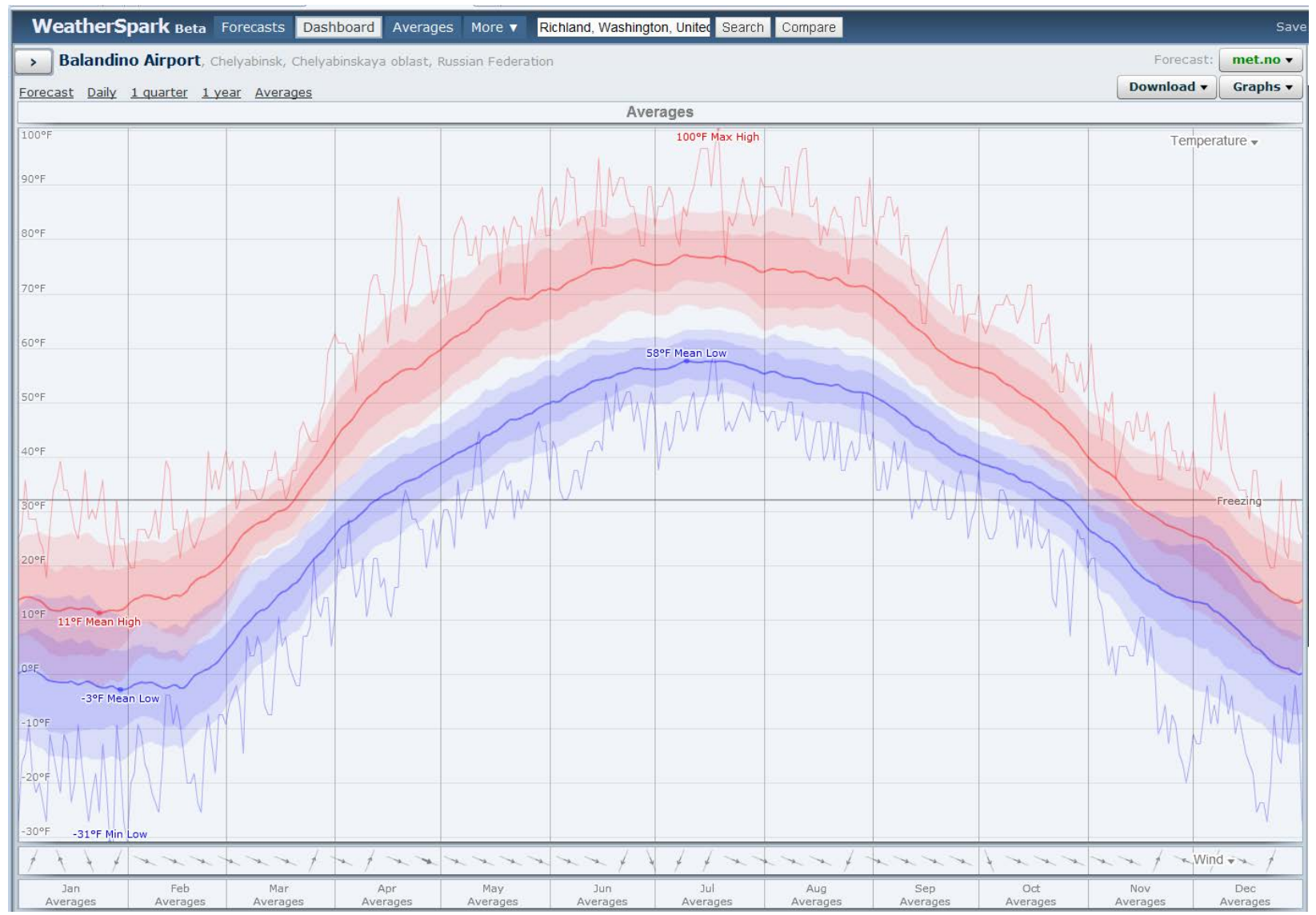

Figure 5.1 Historical temperature data for Balandino Airport, Chelyabinsk, Russian Federation

A suite of library files containing temperatures that trigger changes between animal feeding season dates is required when computing animal product concentrations. Generally, these library files are produced by the utility code FrostpUno. General feeding season dates are available (Mokrov et al. 2007a), section 3 and more information is provided in Appendix B of (Rovny et al. 2009). However, little or no date information is available for any specific year. Thus, the frost dates described in Section 5.1 are used to trigger feeding season changes.

We do note that average monthly temperatures for the Argayash weather station are available (Table A.3.3 of (Drozhko and Khokhryakov 2003)) for 1949 through 1972. Even though we didn't take this approach, the April and October data could be used to shift the overall average dates to account for cooler and warmer months than the overall average. If this approach were used, one would probably reduce the stochastic ranges to a week on either side of the shifted average.

\subsection{ADIETP: Animal Diet Libraries}

A series of library files containing daily animal diet information is required for computing concentrations of animal products in DESCARTES. The library files were generated using the utility program ADIETP. The ADIETP code reads a keyword file and outputs a randomized library containing daily diet information. The runs that generated the library files are identified in Table 5.2. Although the animal 
feeding regimes are allowed to change from year to year, this analysis uses the same feeding regime every year. General animal consumption information is available (Mokrov et al. 2007a), sections 3 and 4. Additional information is provided in Appendix A of (Rovny et al. 2009).

Table 5.2 Keyword Files and Runs for the ADIETP Code

\begin{tabular}{lll}
\hline Animal & Case & Keyword File \\
\hline Poultry & Best Estimate & PoulDietBest.kwd \\
\hline Poultry & Stochastic & PoulDietStoc.kwd \\
\hline Poultry & Mono & PoulDietMono.kwd \\
\hline Beef & Best Estimate & BeefDietBest.kwd \\
\hline Beef & Stochastic & BeefDietStoc.kwd \\
\hline Beef & Mono & BeefDietMono.kwd \\
\hline Goat & Best Estimate & GoatDietBest.kwd \\
\hline Goat & Stochastic & GoatDietStoc.kwd \\
\hline Goat & Mono & GoatDietMono.kwd \\
\hline Milk Cow & Best Estimate & CowDietBest.kwd \\
\hline Milk Cow & Stochastic & CowDietStoc.kwd \\
\hline Milk Cow & Mono & CowDietMono.kwd \\
\hline
\end{tabular}

\subsection{RECIPE: Commercial Leafy Vegetables}

The commercial distribution system for leafy vegetables (defined by which production locations supplied vegetables to consumption locations) is specified in the form of a "recipe" for every year in the simulation. These "recipes" are generated by the utility program RECIPE. The RECIPE code is controlled by a keyword file and outputs a report file and two files for use in the DESCARTES code. The keyword files are identified in Table 5.3.

Table 5.3 Keyword Files for the RECIPE code for Commercial Leafy Vegetables

\begin{tabular}{llll}
\hline Best Estimate Case & Stochastic Case & Mono Case & Year \\
\hline VegRecipe1948Best.kwd & VegRecipe1948Stoc.kwd & VegRecipe1948Mono.kwd & 1948 \\
\hline VegRecipe1949Best.kwd & VegRecipe1949Stoc.kwd & VegRecipe1949Mono.kwd & 1949 \\
\hline VegRecipe1950Best.kwd & VegRecipe1950Stoc.kwd & VegRecipe1950Mono.kwd & 1950 \\
\hline VegRecipe1951Best.kwd & VegRecipe1951Stoc.kwd & VegRecipe1951Mono.kwd & 1951 \\
\hline VegRecipe1952Best.kwd & VegRecipe1952Stoc.kwd & VegRecipe1952Mono.kwd & 1952 \\
\hline VegRecipe1953Best.kwd & VegRecipe1953Stoc.kwd & VegRecipe1953Mono.kwd & 1953 \\
\hline VegRecipe1954Best.kwd & VegRecipe1954Stoc.kwd & VegRecipe1954Mono.kwd & 1954 \\
\hline VegRecipe1955Best.kwd & VegRecipe1955Stoc.kwd & VegRecipe1955Mono.kwd & 1955 \\
\hline VegRecipe1956Best.kwd & VegRecipe1956Stoc.kwd & VegRecipe1956Mono.kwd & 1956 \\
\hline VegRecipe1957Best.kwd & VegRecipe1957Stoc.kwd & VegRecipe1957Mono.kwd & 1957 \\
\hline VegRecipe1958Best.kwd & VegRecipe1958Stoc.kwd & VegRecipe1958Mono.kwd & 1958 \\
\hline VegRecipe1959Best.kwd & VegRecipe1959Stoc.kwd & VegRecipe1959Mono.kwd & 1959 \\
\hline VegRecipe1960Best.kwd & VegRecipe1960Stoc.kwd & VegRecipe1960Mono.kwd & 1960
\end{tabular}




\begin{tabular}{llll}
\hline Best Estimate Case & Stochastic Case & Mono Case & Year \\
\hline VegRecipe1961Best.kwd & VegRecipe1961Stoc.kwd & VegRecipe1961Mono.kwd & 1961 \\
\hline VegRecipe1962Best.kwd & VegRecipe1962Stoc.kwd & VegRecipe1962Mono.kwd & 1962 \\
\hline VegRecipe1963Best.kwd & VegRecipe1963Stoc.kwd & VegRecipe1963Mono.kwd & 1963 \\
\hline VegRecipe1964Best.kwd & VegRecipe1964Stoc.kwd & VegRecipe1964Mono.kwd & 1964 \\
\hline VegRecipe1965Best.kwd & VegRecipe1965Stoc.kwd & VegRecipe1965Mono.kwd & 1965 \\
\hline VegRecipe1966Best.kwd & VegRecipe1966Stoc.kwd & VegRecipe1966Mono.kwd & 1966 \\
\hline
\end{tabular}

According to (Mokrov et al. 2007a), page 20, leafy vegetables were mainly produced by associations of gardeners and in private gardens. Vegetable products from collective farms had only a minor role in consumption for Ozersk residents. However, DESCARTES requires a commercial leafy vegetable distribution system to assign production locations to any garden or farm not located in the same grid cell as the primary residence of the individual. Due to a lack of information, the same production fractions by collective farm are used for commercial leafy vegetables as were used for milk production. In addition, every year after 1956 used the production information for 1956. The spreadsheet Mayak_Recipes.xlsx (see Section 7.1 for further information) can be used to build the keyword files. The CiderF code can select either locally produced or commercial vegetables for a dose calculation.

\subsection{RECIPE: Nodes Supplying Milk to Creameries}

A series of runs of the utility program RECIPE were performed to build libraries of information about which locations (nodes) produced milk for each commercial creamery. In this context, a commercial creamery is equivalent to a collective farm. These files are identified in Table 5.4.

Table 5.4 Keyword Files for the RECIPE code for Creamery Milk Production

\begin{tabular}{llll}
\hline Best Estimate Case & Stochastic Case & Mono Case & Year \\
\hline Cream1948Best.kwd & Cream1948Stoc.kwd & Cream1948Mono.kwd & 1948 \\
\hline Cream1949Best.kwd & Cream1949Stoc.kwd & Cream1949Mono.kwd & 1949 \\
\hline Cream1950Best.kwd & Cream1950Stoc.kwd & Cream1950Mono.kwd & 1950 \\
\hline Cream1951Best.kwd & Cream1951Stoc.kwd & Cream1951Mono.kwd & 1951 \\
\hline Cream1952Best.kwd & Cream1952Stoc.kwd & Cream1952Mono.kwd & 1952 \\
\hline Cream1953Best.kwd & Cream1953Stoc.kwd & Cream1953Mono.kwd & 1953 \\
\hline Cream1954Best.kwd & Cream1954Stoc.kwd & Cream1954Mono.kwd & 1954 \\
\hline Cream1955Best.kwd & Cream1955Stoc.kwd & Cream1955Mono.kwd & 1955 \\
\hline Cream1956Best.kwd & Cream1956Stoc.kwd & Cream1956Mono.kwd & 1956 \\
\hline Cream1957Best.kwd & Cream1957Stoc.kwd & Cream1957Mono.kwd & 1957 \\
\hline Cream1958Best.kwd & Cream1958Stoc.kwd & Cream1958Mono.kwd & 1958 \\
\hline Cream1959Best.kwd & Cream1959Stoc.kwd & Cream1959Mono.kwd & 1959 \\
\hline Cream1960Best.kwd & Cream1960Stoc.kwd & Cream1960Mono.kwd & 1960 \\
\hline Cream1961Best.kwd & Cream1961Stoc.kwd & Cream1961Mono.kwd & 1961 \\
\hline Cream1962Best.kwd & Cream1962Stoc.kwd & Cream1962Mono.kwd & 1962 \\
\hline Cream1963Best.kwd & Cream1963Stoc.kwd & Cream1963Mono.kwd & 1963
\end{tabular}




\begin{tabular}{llll}
\hline Best Estimate Case & Stochastic Case & Mono Case & Year \\
\hline Cream1964Best.kwd & Cream1964Stoc.kwd & Cream1964Mono.kwd & 1964 \\
\hline Cream1965Best.kwd & Cream1965Stoc.kwd & Cream1965Mono.kwd & 1965 \\
\hline Cream1966Best.kwd & Cream1966Stoc.kwd & Cream1966Mono.kwd & 1966 \\
\hline
\end{tabular}

Data needed for this suite of files are provided in Table 2 of (Mokrov et al. 2007a). Also, the map of nodes shown in Figure 2.1 is used to identify which nodes are within the boundaries of each collective farm. In addition, every year after 1966 used the information for 1966. The spreadsheet

Mayak_Recipes.xlsx can be used to build the keyword files.

\subsection{RECIPE: Creameries Supplying Grocery Milk}

A series of runs of the utility program RECIPE are needed to build libraries of information about which creameries (collective farms) contributed to grocery milk consumed at each location. These files are identified in Table 5.5.

Table 5.5 Keyword Files for the RECIPE code for Grocery Milk Recipes

\begin{tabular}{llll}
\hline Best Estimate Case & Stochastic Case & Mono Case & Year \\
\hline MilkDist1948Best.kwd & MilkDist1948Stoc.kwd & MilkDist1948Mono.kwd & 1948 \\
\hline MilkDist1949Best.kwd & MilkDist1949Stoc.kwd & MilkDist1949Mono.kwd & 1949 \\
\hline MilkDist1950Best.kwd & MilkDist1950Stoc.kwd & MilkDist1950Mono.kwd & 1950 \\
\hline MilkDist1951Best.kwd & MilkDist1951Stoc.kwd & MilkDist1951Mono.kwd & 1951 \\
\hline MilkDist1952Best.kwd & MilkDist1952Stoc.kwd & MilkDist1952Mono.kwd & 1952 \\
\hline MilkDist1953Best.kwd & MilkDist1953Stoc.kwd & MilkDist1953Mono.kwd & 1953 \\
\hline MilkDist1954Best.kwd & MilkDist1954Stoc.kwd & MilkDist1954Mono.kwd & 1954 \\
\hline MilkDist1955Best.kwd & MilkDist1955Stoc.kwd & MilkDist1955Mono.kwd & 1955 \\
\hline MilkDist1956Best.kwd & MilkDist1956Stoc.kwd & MilkDist1956Mono.kwd & 1956 \\
\hline MilkDist1957Best.kwd & MilkDist1957Stoc.kwd & MilkDist1957Mono.kwd & 1957 \\
\hline MilkDist1958Best.kwd & MilkDist1958Stoc.kwd & MilkDist1958Mono.kwd & 1958 \\
\hline MilkDist1959Best.kwd & MilkDist1959Stoc.kwd & MilkDist1959Mono.kwd & 1959 \\
\hline MilkDist1960Best.kwd & MilkDist1960Stoc.kwd & MilkDist1960Mono.kwd & 1960 \\
\hline MilkDist1961Best.kwd & MilkDist1961Stoc.kwd & MilkDist1961Mono.kwd & 1961 \\
\hline MilkDist1962Best.kwd & MilkDist1962Stoc.kwd & MilkDist1962Mono.kwd & 1962 \\
\hline MilkDist1963Best.kwd & MilkDist1963Stoc.kwd & MilkDist1963Mono.kwd & 1963 \\
\hline MilkDist1964Best.kwd & MilkDist1964Stoc.kwd & MilkDist1964Mono.kwd & 1964 \\
\hline MilkDist1965Best.kwd & MilkDist1965Stoc.kwd & MilkDist1965Mono.kwd & 1965 \\
\hline MilkDist1966Best.kwd & MilkDist1966Stoc.kwd & MilkDist1966Mono.kwd & 1966 \\
\hline
\end{tabular}

This application treated creameries (collective farms) as milk suppliers to grocery stores. Data needed for this suite of files is provided in Table 2 of (Mokrov et al. 2007a). Grocery milk was only computed for consumption at Ozersk. Residents of the city were assumed to live at one of five dose model nodes (390, 
391, 392, 414, or 415). In addition, every year after 1966 used the production information for 1966. The spreadsheet Mayak_Recipes.xlsx can be used to build the keyword files.

\subsection{RECIPE: Feeding Regimes Active for Creamery Milk}

A run of the utility program RECIPE is needed to build a library of information about which cow feeding regimes were active at nodes that supply commercial milk to creameries. The required files are identified in Table 5.6.

Table 5.6 Keyword Files and Runs for the RECIPE Code

\begin{tabular}{ll}
\hline Purpose & Keyword File \\
\hline Best Estimate Case & FregimesBest.kwd \\
\hline Stochastic Case & FregimesStoc.kwd \\
\hline Mono Case & FregimesMono.kwd \\
\hline
\end{tabular}

Bovine feeding practices varied slightly by location and year. See section 4 in (Mokrov et al. 2007a) and also (Rovny et al. 2009). However, this variation in feeding practices was slight enough, given the animal food products computed by DESCARTES, that only one feeding regime was identified. The other three possible animal feeding regimes that can be used in the DESCARTES and CiderF codes were not utilized.

\subsection{Runs of the DESCARTES Code}

Three runs of the DESCARTES code were made. The keyword files for these runs are identified in Table 5.7. Each of these runs produces a suite of 27 "media" files containing concentrations in a variety of media that will be used in the CiderF code. The preprocessors identified in Sections 5.1 through 5.6 prepared multiple input files used in each run of the DESCARTES code.

Table 5.7 Keyword Files and Runs for the DESCARTES Code

\begin{tabular}{lll}
\hline Purpose & Keyword File & Data Package Directory \\
\hline Best Estimate Case & Best.kwd & $\backslash$ Mayak\Best \\
\hline Stochastic Case & Stochastic.kwd & $\backslash$ Mayak\Stochastic \\
\hline Mono Case & Mono.kwd & $\backslash$ Mayak\Mono \\
\hline
\end{tabular}




\subsection{Runs of the CiderF Code}

The general approach for the dose calculations is to define a handful of reference individuals and perform runs to show the dose distributions by year and total. The CiderF code was used to generate doses for the time period of interest.

The CiderF code can generate doses in two different modes. The individual mode allows the user to calculate doses for one or more individuals. The individual lives in user specified locations and eats either a reference diet or a user specified special diet. The primary output of the code is the dose to the individual. The second mode, the map mode, applies the same characteristics to an individual living at every single node in the dose domain. For example, one can obtain the dose to a five year old child using rural lifestyle assumptions at every node in the domain.

The keyword inputs for CiderF are divided into two files. One file (the factors keyword file) contains information that does not vary even if doses are calculated for more than one individual. The other file (case keyword file) contains the minimal set of keywords needed to define individual dose cases or a map case. Information that does not change by dose case was collected in the Mayak_ref_Diet.xlsx spreadsheet to facilitate generating keyword inputs for the codes.

\subsection{Reference Information}

Dietary information and recommended lifestyle and exposure information is provided in (Rovny et al. 2009). Although that document contains a large amount of information about the habits and activities of individuals living near Ozersk in the past, no specific dose code was identified before the information was collected. Thus, some information, especially dietary information, was not in the format needed by the CiderF code.

Entries in the reference keyword files include the following types of information:

- Specific consumption sources and rates for each reference diet

- Breathing rates

- Fraction of time spent outdoors

- Dose factors for external, inhalation and ingestion exposure

- Holdup times by food categories

- Food processing loss fractions, and

- Dry to wet ratios for food types.

The four "reference" factors keyword files identified in Table 6.1 were prepared for the CiderF code using the information in the spreadsheet Mayak_ref_Diet.xlsx. These four files are located in directory $\backslash$ Mayak $\backslash$ Kwd $\backslash$ RefDiet of the data package. There are a large number of keyword entries in each of these files. The relationship between the specific keywords and the spreadsheet entries used to develop the keywords are captured in comments in the keyword file.

The best estimate, stochastic and mono cases are optimized for local vegetable consumption. In addition, rural lifestyles default to local cow milk production and urban lifestyles default to grocery milk from 
collective farms. The worker case defaults to locally produced cow milk and consumption of local vegetables. In the default data sets, commercial milk products and commercial leafy vegetables are available only in Ozersk. Specifically, this means that commercial products are only available for people living at nodes 390, 391, 392, 414 and 415. These locations are identified in Figure 2.1 or the file “MayakGridNumbered.kmz” can be opened in Google Earth to zoom in on the identified locations.

Table 6.1 Reference Diet, Lifestyle and Exposure Factors Keyword Files for CiderF Code

\begin{tabular}{ll}
\hline Purpose & Keyword File \\
\hline Best Estimate Case & MayakFactorsBest.kwd \\
\hline Stochastic Case & MayakFactorsStoc.kwd \\
\hline Mono Case & MayakFactorsMono.kwd \\
\hline Stochastic Worker Case & MayakFactorsWorkerStoc.kwd \\
\hline
\end{tabular}

The CiderF dose code uses the concept of reference and special diets. A series of reference diets are defined for a variety of ages, lifestyle activities and residence type. All runs of the CiderF code use the reference diets unless the consumption pattern is modified by a case-specific special diet. A total of 14 reference diets are defined. The mapping of the reference diets to lifestyle activities is provided in Table 6.2.

Table 6.2 Reference Diet Definitions by Lifestyle Categories

\begin{tabular}{ll}
\hline Diet ID & Lifestyle Categories \\
\hline RefDiet001 & Child, 0 to 6 months \\
\hline RefDiet002 & Child, >6 months to 2 year \\
\hline RefDiet003 & Child, rural, >2 years to 7 years \\
\hline RefDiet004 & Child, urban, $>2$ years to 7 years \\
\hline RefDiet005 & Child, rural, $>7$ years to 12 years \\
\hline RefDiet006 & Child, urban, >7 years to 12 years \\
\hline RefDiet007 & Child, rural, $>12$ years to 17 years \\
\hline RefDiet008 & Child, urban, $>12$ years to 17 years \\
\hline RefDiet009 & Adult, rural \\
\hline RefDiet010 & Adult, urban \\
\hline RefDiet011 & Suckling child, 0 to 6 months \\
\hline RefDiet012 & Suckling child, >6 months to 2 year \\
\hline RefDiet013 & Adult, rural, pregnant/nursing female \\
\hline RefDiet014 & Adult, urban, pregnant/nursing female \\
\hline
\end{tabular}

\subsection{Example Dose Map Runs}

A run of the CiderF code using best estimate parameters was performed in map mode for a rural child that was five years old. Separate map cases in the run modeled a child that was 5 in 1948, a child that was 5 in 1949, and a child that was 5 for every year from 1950 through 1972. The keyword files for this run are identified in Table 6.3. Also identified is the keyword file for a graphics postprocessor named CiderView. The postprocessor produces a KML file of dose contours suitable for viewing in Google Earth. These files are located in the directory $\backslash$ Mayak $\backslash$ Best $\backslash$ Cases $\backslash$ Child5 in the data package. 
Table 6.3 Keyword Files for the Best Estimate Map Run of the CiderF Code

\begin{tabular}{ll}
\hline Purpose & Keyword File \\
\hline CiderF Keyword File & Child5Best.kwd \\
\hline Reference Information Keyword File & MayakFactorsBest.kwd \\
\hline CiderView Keyword File & Child5View.kwd \\
\hline
\end{tabular}

An example output of the CiderView code (as visualized in Google Earth) for the best estimate run is provided in Figure 6.1 for a child who is 5 years old in 1949. The values plotted are thyroid dose (rad). All of the release facilities are in the dark colored region that is located southeast of Ozersk. The map domain in the figure uses only the rectangular portion of the dose grid.

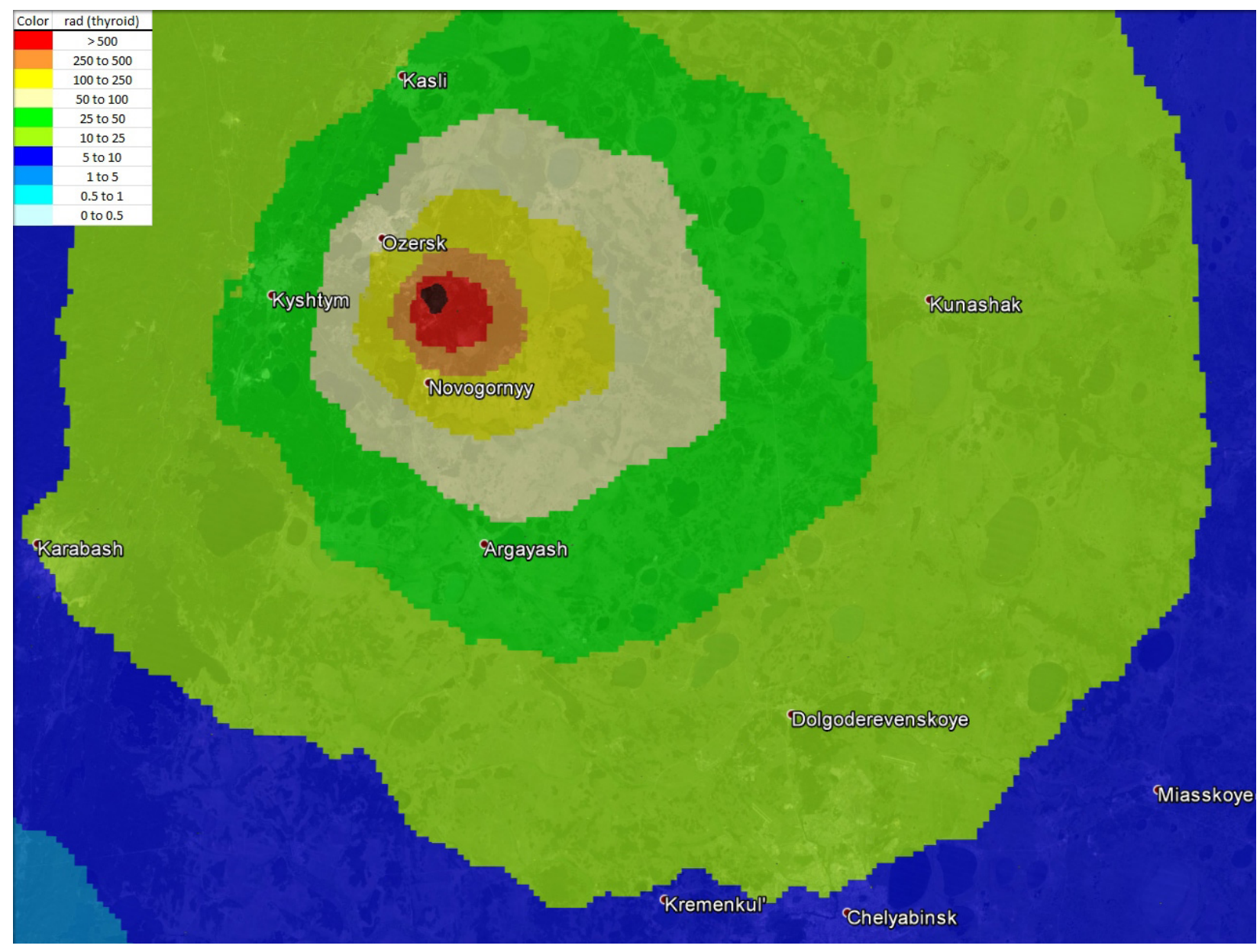

Figure 6.1 Best Estimate Thyroid Dose (rad) for a Rural 5-Year-Old Child in 1949

A run of the dose code using stochastic parameters was also performed in map mode for a rural child that was five years old for every year from 1950 through 1972. The keyword files for this run are identified in Table 6.4. Also identified is the keyword file for the graphics postprocessor CiderView. 
Table 6.4 Keyword Files for the Stochastic Map Run for a Rural Child

\begin{tabular}{ll}
\hline Purpose & Keyword File \\
\hline CiderF Keyword File & Child5Stoc.kwd \\
\hline Reference Information Keyword File & MayakFactorsStoc.kwd \\
\hline CiderView Keyword File & Child5View.kwd \\
\hline
\end{tabular}

An example output of the CiderView code for a stochastic run is provided in Figure 6.2 for a child who is 5 years old in 1949. The values plotted are thyroid dose (rad). The values in the top pane of the figure show the $1 \%$ level of the stochastic doses at each location. The values in the bottom pane show the $99 \%$ level of the stochastic doses at each location.

Another run of the CiderF dose code using stochastic parameters was performed in map mode for a male worker. The worker was assumed to be born in 1928 and the computed doses are for inhalation exposure for the entire time period of 1948 through 1972. The keyword files for this run are identified in Table 6.5. Also identified is the keyword file for the graphics postprocessor CiderView. The data files for this case are located in the $\backslash$ Mayak $\backslash$ Stochastic $\backslash$ Maps $\backslash$ Worker directory of the data package.

Table 6.5 Keyword Files for the Stochastic Map Run for a Worker

\begin{tabular}{ll}
\hline Purpose & Keyword File \\
\hline CiderF Keyword File & WorkerBYCowStoc.kwd \\
\hline Reference Information Keyword File & MayakFactorsWorkerStoc.kwd \\
\hline CiderView Keyword File & WorkerBYCowView.kwd \\
\hline
\end{tabular}

An example output of the CiderView code for the worker case is provided in Figure 6.3. The values plotted are the median thyroid dose (Gy) due to inhalation, summed over the years 1948 through 1972. This particular plot shows the inhalation doses for the central region of the grid as well as the portion of the domain that follows the path of the Techa River to the northeast. 

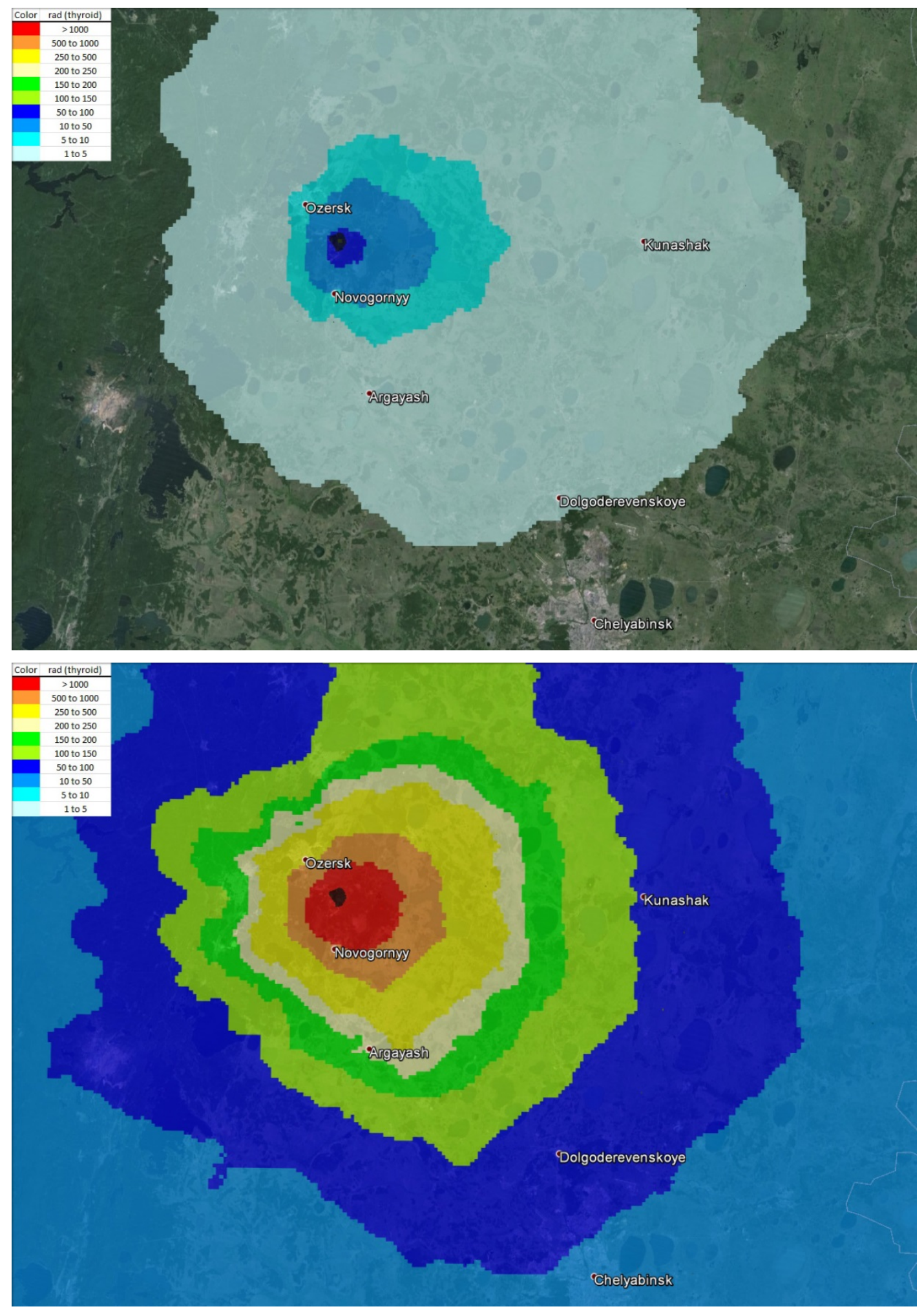

Figure 6.2 Stochastic Thyroid Dose (rad) for a Rural 5 Year Old Child in 1949 (1\% level in top pane, 99\% level in bottom pane) 


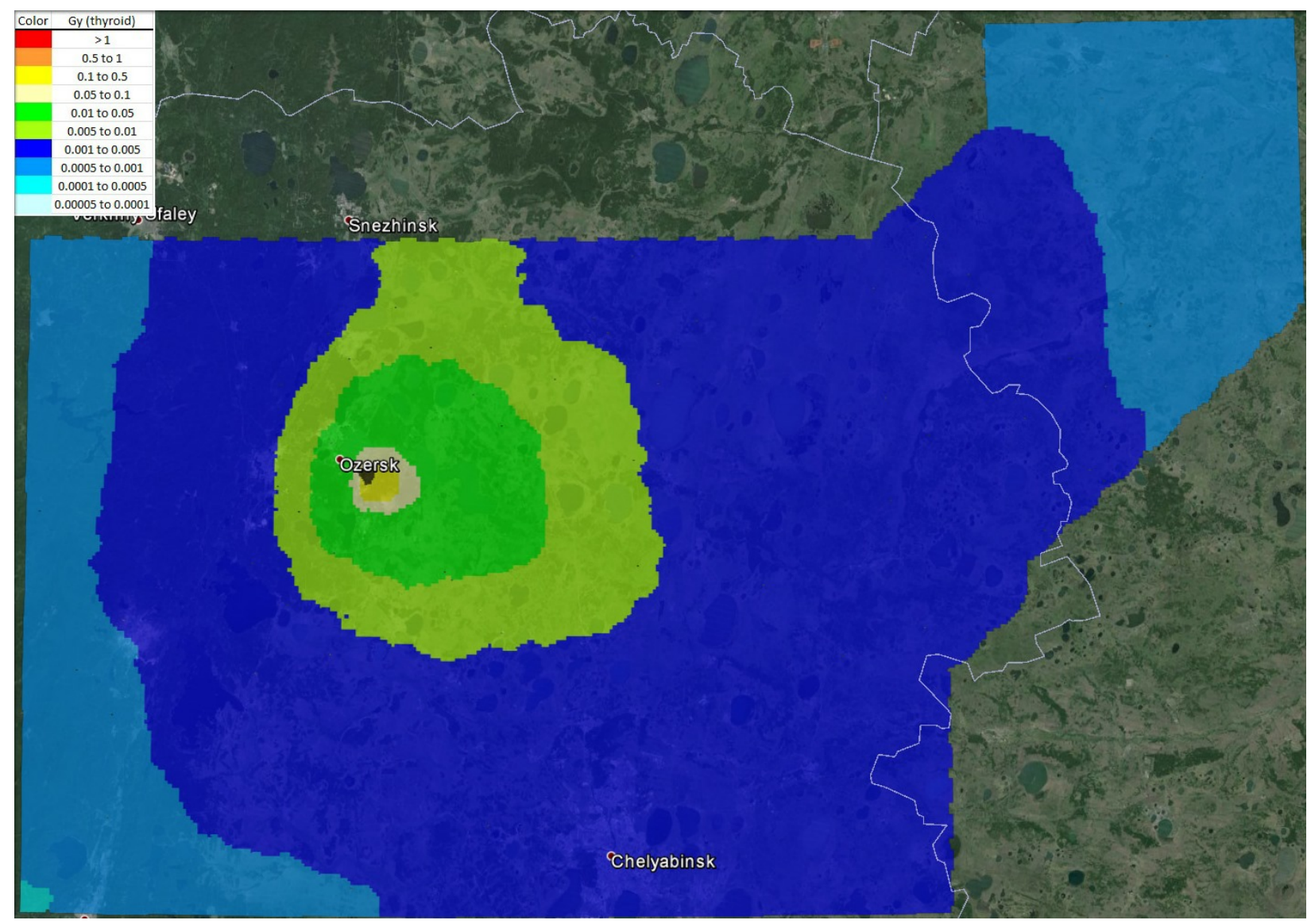

Figure 6.3 Median Thyroid Dose (Gy) for a Local Worker Due to Inhalation Totaled over the Years 1948 through 1972

\subsection{Example Individual Dose Runs}

Three example individual case runs were performed for an individual living in Ozersk. These three runs all model a five year old child using rural lifestyle assumptions. The runs differ in how they treat the atmospheric runs and the parameters describing the individual. The primary features of the three cases identified in Table 6.6 are the following:

- Best Estimate Case: Single realization case using best estimate values for release, accumulation, and individual parameters along with averaged atmospheric transport results.

- Stochastic Case: Two-hundred fifty realization run using stochastic descriptions for release, accumulation, and individual parameters along with stochastic atmospheric transport results.

- Mono Case: Two-hundred fifty realization run using stochastic descriptions for release and stochastic atmospheric transport results but best estimates for environmental accumulation and individual dose parameters. 
Table 6.6 Keyword Files for Map Cases Using the CiderF Code

\begin{tabular}{lllll}
\hline Purpose & Release & Atmospheric & Individual & Reference Keyword File \\
\hline Best Estimate Case & Stochastic & Averaged & Best Estimate & MayakFactorsBest.kwd \\
\hline Stochastic Case & Stochastic & Stochastic & Stochastic & MayakFactorsStoc.kwd \\
\hline Mono Case & Stochastic & Stochastic & Best Estimate & MayakFactorsMono.kwd \\
\hline
\end{tabular}

As an example calculation, the percent of the variability in the estimated thyroid doses that can be attributed to the variability in release and atmospheric transport for a 5 year old child living in Ozersk in 1950 is shown in Table 6.7. The percent values listed in the first column of the table are based on the ratio of standard deviations of dose by pathway. The divisor uses doses from the full stochastic run (Stochastic Case). The numerator uses doses from the Mono case, thus release and atmospheric transport are stochastic but environmental accumulation and individual exposure parameters are set at fixed best estimate values. Also included is the mean dose from the stochastic case. For the mono case, the child eats 80 grams of beef a day and drinks $0.425 \mathrm{~L}$ of fresh milk per day.

Table 6.7 Percent of Variability in Thyroid Dose to a 5 Year Old Child Living in Ozersk in 1950 Attributable to Variability in Facility Releases and Atmospheric Transport

\begin{tabular}{llll}
\hline $\begin{array}{l}\text { Percent of } \\
\text { Variability }\end{array}$ & $\begin{array}{l}\text { Mean } \\
\text { Dose (rad) }\end{array}$ & $\begin{array}{l}\text { Percent of } \\
\text { Total Dose }\end{array}$ & Dose Pathway \\
\hline $47.4 \%$ & 33.57 & NA & Total \\
\hline $63.5 \%$ & 0.001413 & $0.004 \%$ & External \\
\hline $18.4 \%$ & 0.4150 & $1.236 \%$ & Inhalation \\
\hline $53.2 \%$ & 2.902 & $8.64 \%$ & Beef ingestion \\
\hline $51.2 \%$ & 0.09373 & $0.279 \%$ & Leafy vegetable ingestion \\
\hline $62.4 \%$ & 0.01527 & $0.045 \%$ & Other vegetable ingestion \\
\hline $45.7 \%$ & 0.08590 & $0.256 \%$ & Fruit ingestion \\
\hline $84.4 \%$ & 0.004783 & $0.014 \%$ & Grain ingestion \\
\hline $45.2 \%$ & 0.001326 & $0.004 \%$ & Poultry ingestion \\
\hline $36.1 \%$ & 0.2824 & $0.841 \%$ & Eggs ingestion \\
\hline $48.6 \%$ & 29.77 & $88.68 \%$ & Milk Ingestion \\
\hline
\end{tabular}

An example comparison of the best estimate run and the stochastic run for a five year old male child living in Ozersk in different years is provided in Figure 6.4. The boxes in the plots show the 25\%, median and $75 \%$ levels of the stochastic doses. The cross marks on the tails shows the $5 \%$ and $95 \%$ levels of dose. The tails extend to the $1 \%$ and $99 \%$ levels of dose. The triangles show the minimum and maximum levels of dose. The red dots are the corresponding results from the best estimate case. As expected, the dose values follow the trend of the annual releases shown in Figure 4.2. The spreadsheet named Child5_Ozersk.xlsm was used to compile the plot data. The macro named BuildPlot was used to create the curves because Excel does not provide a function to develop this type of plot. 


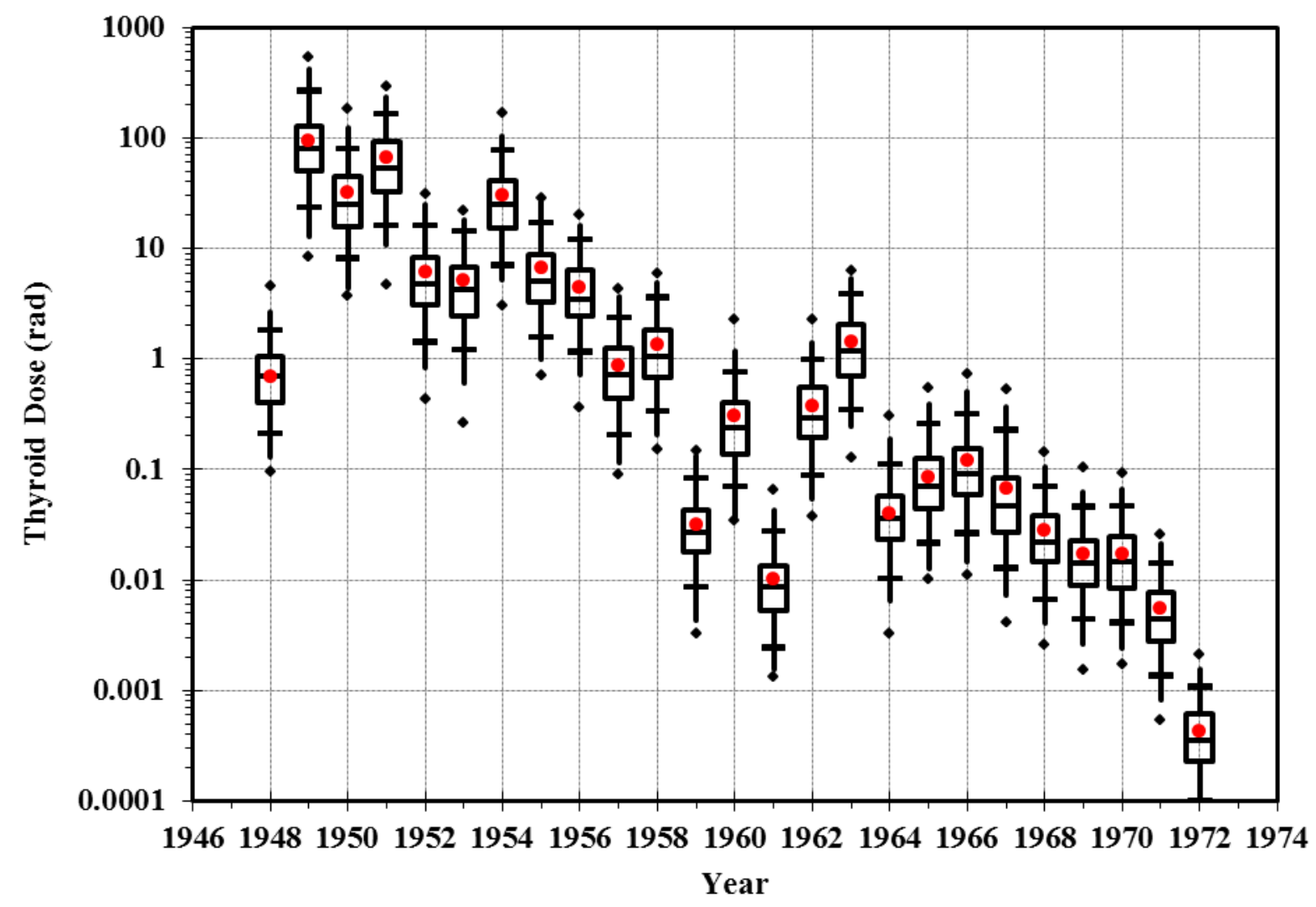

Figure 6.4 Annual Thyroid Dose for a 5 Year Old Male Child Living in Ozersk

Another example calculation examined the variability in the full stochastic case for a 5-year old male child living in Ozersk in 1949. In this case, a regression analysis determined that the variability in the internal dose factor accounted for $63 \%$ of the variability in the total thyroid dose. The scatterplot in Figure 6.5 shows the effect of the internal dose factor on the total dose. The spreadsheet named Child5_Sensitivity.xlsx was used to perform the regression and compile the plot data. For 1949, about $85.5 \%$ of the dose is from milk ingestion and another $10.8 \%$ is from beef ingestion. Total ingestion is $98.275 \%$ of the dose, inhalation is $1.717 \%$, and external is the remaining $0.007 \%$.

We expanded the regression analysis on the doses for 1949 to include the total yearly stochastic releases from A reactor and B plant. Including these variables causes the $\mathrm{R}^{2}$ to increase to $65.1 \%$ and the included variable coefficient is statistically significant. Thus, the variation in annual releases adds to the explanation of total variability. If the speciation fractions (particulate and elemental) are included in the regression setting, the R-square goes up to $68.1 \%$ and both coefficients are statistically significant. It seems likely that the variability in the meteorological data is the remaining big driver in variability. Unfortunately, there isn't any simple way to describe that variability for use in a regression analysis. 


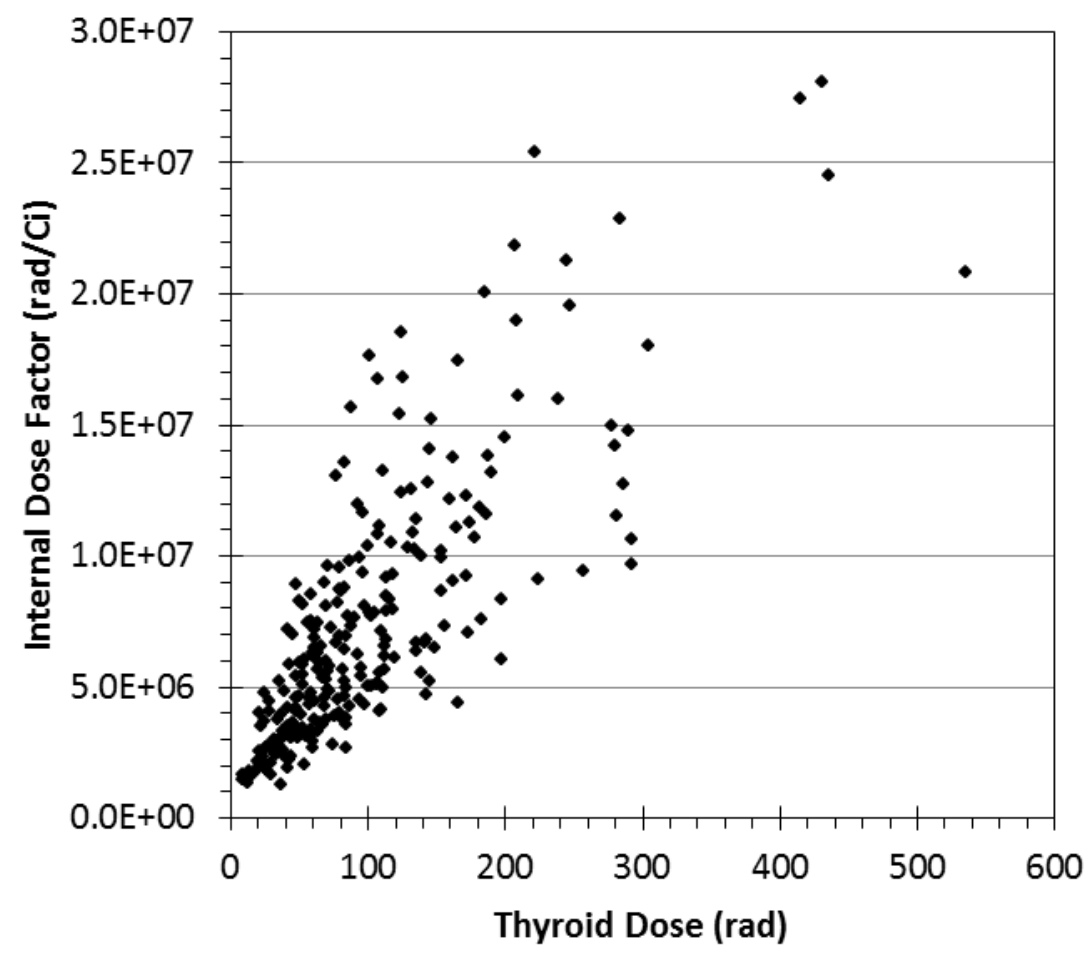

Figure 6.5 Scatterplot of Thyroid Dose to a 5 Year Old Child Living in Ozersk in 1949 against the Internal Dose Conversion Factor 


\subsection{Data Package}

A data package has been prepared to accompany this document. The data package contains outputs of the AirCombGrid Code (see Table 1.1) and inputs and outputs for all subsequent codes in the modeling sequence. In addition, executable codes (Windows 64-bit versions) of the codes are provided. The purpose of the data package is to provide the input files and executable codes necessary to recreate all of the results given in this document, subsequent to the execution of the AirGridComb code. The data package is provided in a directory structure as follows:

Mayak: Top level directory

Best: Information for best estimate runs, including inputs and outputs of the DESCARTES code

AirCombGrid: Keyword inputs and output files from the AirCombGrid code

Cases: Directory structure for individual cases

Maps: Directory structure for map cases

Codes: Executable codes

DataReports: PDF's of the milestone reports used to develop the input data for the environmental accumulation and dose codes

Kwd: Information and spreadsheets for preparing keywords

AnimalDiets: Keywords and data files for animal diets

FrostpUno: Keywords and data files for frost dates

Recipe: Keywords and data files for commercial leafy vegetables, grocery milk and creamery milk production

RefDiet: Keywords files for reference diets

Mono: Information for the "mono" runs, including inputs and outputs of the DESCARTES code.

Cases: Directory structure for individual cases

Spreadsheets: Copies of Excel spreadsheets containing information to assist with preparing code inputs and analyzing output results

Stochastic: Information for the stochastic runs, including inputs and outputs of the DESCARTES code

AirCombGrid: Keyword inputs and output files from the AirCombGrid code

Cases: Directory structure for individual cases

Maps: Directory structure for map cases

The data package (in the directory Mayak) contains 2,461 files in 24 folders and uses $19.1 \mathrm{~Gb}$ of disk space.

\subsection{Spreadsheets}

Several spreadsheets are used to facilitate preparing inputs in the format needed by the computer codes. Other spreadsheets are used to analyze run results. These spreadsheets are located in the MayakıSpreadsheets directory and contain the following information:

Best_Release.xlsx - This spreadsheet contains inventory data output by the AirCombGrid for the best estimate case. This spreadsheet was used to develop Figure 4.1and Figure 4.2. 
Child5_Ozersk.xlsm - This macro-enabled spreadsheet contains information from CiderF best estimate, stochastic and mono runs used to build the box and whisker plot given in Figure 6.4. This spreadsheet was also used to produce Table 6.7.

Child5_Sensitivity.xlsm - This spreadsheet contains information on the sensitivity analysis for a child in Ozersk in 1949 discussed in Section 6.3 and it was used to generate the scatterplot in Figure 6.5.

Grid.xlsx - This spreadsheet contains information used to build the NODE keywords for the 618 nodes in the dose domain.

Legend.xlsm - This macro-enabled spreadsheet generates KMLCOLOR and KMLLEGEND keywords for the AirCombGridView and CiderView codes. It also generates the associated legend that can be saved into a graphics file.

Mayak_Recipes.xlsx - This spreadsheet collects information for production and distribution "recipes" of commercial leafy vegetables and commercial milk (collective farms and grocery stores). Best estimate and stochastic keywords are generated for use in the RECIPE code. Keywords for different years are generated by entering a different row number in cell K1 of the "Dairy-Cream”, “Grocery” and "Leafy” sheets.

Mayak_Ref_Diet.xlsx - This spreadsheet collects the information on reference diets and the other data needed for the CiderF Factors Keyword file (see Section 8.7 of the CiderF user's Guide). Best estimate and stochastic keywords are generated for use in the CiderF code.

Release_Keywords.xlsx - This spreadsheet collects the information on the monthly releases from the eight facilities. Best estimate and stochastic keywords are generated for use in the AirCombGrid code.

Release_Points.xlsx - This spreadsheet collects information on the release points and operational periods for the eight facilities.

Stochastic_Release.xlsx - This spreadsheet contains inventory data output by the AirCombGrid for the stochastic case. This spreadsheet was used to develop Table 4.2.

\subsection{Code Execution}

Many of the subdirectories in the data package contain batch files named "run.bat". These batch files can be executed by double clicking on them. If the data package is copied to a location where Mayak is the top level directory on the drive, then the run.bat files can generally be executed without modification. One exception is that some file names in the keyword files for AirCombGridView and CiderView must be changed to fully resolved path names so Google Earth can find the files. The batch files will generate the input library files for DESCARTES and CiderF and also execute these two codes. 


\subsection{References}

Anspaugh, LR, and BA Napier. 2009. Minor Parameters Needed for Individual-Dose Calculations: Final Report for Tasks 7.1, 7.2, 8.1, 8.2, 9.1, 9.2, and 9.3, PNNL-18917, Pacific Northwest National Laboratory, Richland, Washington. http://www.pnl.gov/main/publications/external/technical_reports/PNNL-18917.pdf.

Draxler, RR, and GD Hess. 1998. "An Overview of the HYSPLIT_4 Modeling System of Trajectories, Dispersion, and Deposition." Aust. Meteor. Mag. 47:295-308.

Draxler, RR, B Stunder, G Rolph, A Stein, and A Taylor. 2012. HYSPLIT4 User's Guide, Air Resources Laboratory, National Oceanic and Atmospheric Administration (NOAA), Silver Spring, MD. http://www.arl.noaa.gov/documents/reports/hysplit_user_guide.pdf.

Drozhko, EG, and EG Khokhryakov. 2003. Reconstruction of Ozyorsk Population Exposure Doses Due to Iodine-131 Atmospheric Releases from Spent Nuclear Fuel Reprocessing, JCCRER Project 1.4, Ozersk, Russia.

Eslinger, PW, KS Lessor, and SJ Ouderkirk. 1994. User Instructions for the CIDER Dose Code, PNWD2252 HEDR, Battelle, Pacific Northwest Laboratories, Richland, Washington.

DOI:http://dx.doi.org/10.2172/10154408.

Eslinger, PW, and BA Napier. 2013. User Instructions for the CiderF Individual Dose Code and Associated Utility Codes, PNNL-22699, Pacific Northwest National Laboratory, Richland, Washington. http://www.pnl.gov/main/publications/external/technical_reports/PNNL-22699.pdf.

Farris, WT, BA Napier, PW Eslinger, TA Ikenberry, DB Shipler, and CJ Simpson. 1994. Atmospheric Pathway Dosimetry Report, 1944-1992, PNWD-2228 HEDR, Battelle, Pacific Northwest Division, Richland, Washington.

GDAS. 2012. Global Data Assimilation System Archive. Air Resources Laboratory, National Oceanic and Atmospheric Administration. Accessed on April 2, 2012, at ftp://arlftp.arlhq.noaa.gov/pub/archives/gdas1/

Google. 2013. Google Earth. Accessed on May 16, 2013, at http://www.google.com/intl/en/earth/index.html

Miley, TB, PW Eslinger, WE Nichols, KS Lessor, and SJ Ouderkirk. 1994. User Instructions for the DESCARTES Environmental Accumulation Code, PNWD-2251 HEDR, Battelle, Pacific Northwest Laboratories, Richland, Washington. DOI:http://dx.doi.org/10.2172/10154088.

Mokrov, YG, LR Anspaugh, and BA Napier. 2004. Reconstruction of Dose to the Residents of Ozersk from Operation of the Mayak Production Association: 1948-2002 (Final Report on the Feasibility Study for Project 1.4), US-Russian Joint Coordinating Committee on Radiation Effects Research Project 1.4, Ozersk, Russia.

Mokrov, YG, and YG Beregich. 2006. Milestone 2b: Selection of Atmospheric Transport Model and Transfer and Installation of the Selected Atmospheric Dispersion Model to Mpa, US-Russian Joint Coordinating Committee on Radiation Effects Research Project 1.4, Ozersk, Russia. 
Mokrov, YG, VZ Martyushov, PM Stukalov, IA Ivanov, and ES Levunina. 2007a. Milestone 8: Production and Delivery of Food to Ozersk, US-Russian Joint Coordinating Committee on Radiation Effects Research Project 1.4, Ozersk, Russia.

Mokrov, YG, VT Khruchsh, AS Bakurov, GP Shein, and GM Aksyonov. 2007b. Milestone 3: Determination of Quantitative Parameters for the Iodine-131 Model for Transfer to Vegetation, Farm Animal Products, and Agricultural Products, US-Russian Joint Coordinating Committee on Radiation Effects Research Project 1.4, Ozersk, Russia.

Mokrov, YG, AV Lyzhkov, VA Muzrukov, NP Pyatin, SI Rovny, LR Anspaugh, and BA Napier. 2008a. Milestone 7: Reconstruction of Atmospheric Releases of I-131 from Mayak Radiochemical Plant Stacks for the Period from 1948 to 1970, Part 2: Results of the Reconstruction of ${ }^{131}$ I Releases from the Stacks of the Reactors and Radiochemical Plants, US-Russian Joint Coordinating Committee on Radiation Effects Research Project 1.4, Ozersk, Russia.

Mokrov, YG, VZ Martyushov, PM Stukalov, TA Antonova, IA Ivanov, SI Rovny, LR Anspaugh, and BA Napier. 2008b. Milestone 6: Changes in Population Food Ration and Demographic Parameters for Ozersk in 1948-2002. Historical Age-Dependent Food-Consumption Rates, US-Russian Joint Coordinating Committee on Radiation Effects Research Project 1.4, Ozersk, Russia.

Mokrov, YG, SI Rovny, DA Beregich, LR Anspaugh, and BA Napier. 2009. Milestone 10, Part 2: Reconstruction of External Dose Caused by Atmospheric Releases of Noble Radioactive Gases from the Stacks of the Mayak Reactors in 1948-1989., US-Russian Joint Coordinating Committee on Radiation Effects Research Project 1.4, Ozersk, Russia.

Napier, BA, LR Anspaugh, YG Mokrov, and SI Rovny. 2008. Milestone 4: Evaluation of Chemical Forms of ${ }^{131}$ I Involved in Atmoispheric Transport, US-Russian Joint Coordinating Committee on Radiation Effects Research Project 1.4, Ozersk, Russia.

Ramsdell Jr., JV, CA Simonen, and KW Burk. 1994. Regional Atmospheric Transport Code for Hanford Emission Tracking (RATCHET), PNWD-2224 HEDR, Battelle, Pacific Northwest Laboratories, Richland, Washington. DOI:http://dx.doi.org/10.2172/10121918.

Rovny, SI, YG Mokrov, PM Stukalov, DA Beregich, II Teplyakov, LR Anspaugh, and BA Napier. 2009. Methods for Calculating Thyroid Doses to the Residents of Ozersk Due to ${ }^{131}$ I Releases from the Stacks of the Mayak Production Association, PNNL-18916, Pacific Northwest National Laboratory, Richland, Washington. http://www.pnl.gov/main/publications/external/technical_reports/PNNL-18916.pdf.

Snyder, SF, WT Farris, BA Napier, TA Ikenberry, and RO Gilbert. 1994. Parameters Used in the Environmental Pathway and Radiological Dose Modules (DESCARTES, CIDER and CRD Codes) of the Hanford Environmental Dose Reconstruction Integrated Codes (HEDRIC). PNWD-2023 HEDR, Rev. 1, Battelle, Pacific Northwest Laboratories, Richland, Washington. DOI:http://dx.doi.org/10.2172/10158073.

WeatherSpark. 2012. Weather: Balandino Airport, Chelyabinsk, Chelyabinskaya Oblast, Russian Federation. Accessed on November 12, 2012, at http://weatherspark.com/\#!dashboard;ws=33856 


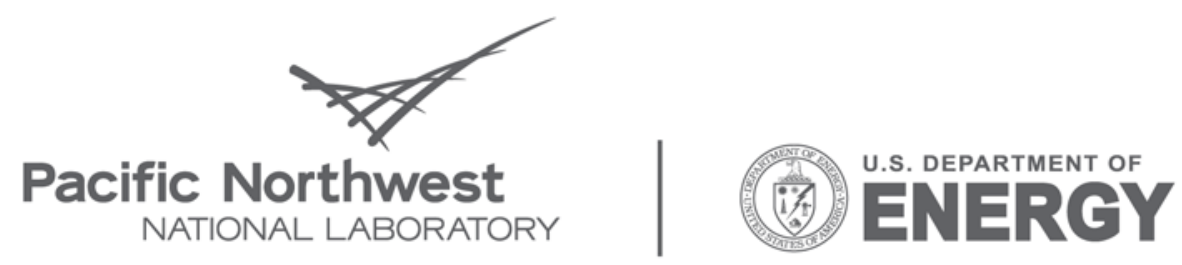

Proudly Operated by Battelle Since 1965

902 Battelle Boulevard

P.O. Box 999

Richland, WA 99352

1-888-375-PNNL (7665)

www.pnl.gov 